\title{
ARTICLE The increased protection and pathology in Mycobacterium tuberculosis-infected IL-27R-alpha-deficient mice is supported by IL-17A and is associated with the IL-17A-induced expansion of multifunctional $\mathrm{T}$ cells
}

\author{
Hanna Erdmann ${ }^{1,2}$, Jochen Behrends ${ }^{1,3}$, Kristina Ritter ${ }^{1}$, Alexandra Hölscher ${ }^{1}$, Johanna Volz ${ }^{1}$, Ida Rosenkrands ${ }^{4}$ and \\ Christoph Hölscher ${ }^{1,2}$
}

\begin{abstract}
During Mycobacterium tuberculosis (Mtb) infection, mice lacking the IL-27R exhibit lower bacterial burdens but develop an immunopathological sequelae in comparison to wild-type mice. We here show that this phenotype correlates with an enhanced recruitment of antigen-specific CCR6 ${ }^{+} \mathrm{CD}^{+} \mathrm{T}$ cells and an increased frequency of IL-17A-producing CD4 ${ }^{+} \mathrm{T}$ cells. By comparing the outcome of Mtb infection in C57BL/6, IL-27R-deficient and IL-27R/IL-17A-double deficient mice, we observed that both the increased protection and elevated immunopathology are supported by IL-17A. Whereas IL-17A neither impacts the development of Tr1 cells nor the expression of PD1 and KLRG1 on T cells in IL-27R-deficient mice during infection, it regulates the presence of multifunctional T-cells in the lungs, co-expressing IFN- $\gamma$, IL-2 and TNF. Eventually, IL-17A supports Cxcl9, Cxcl10 and Cxcl13 expression and the granulomatous response in the lungs of infected IL-27R-deficient mice. Taken together, IL-17A contributes to protection in Mtb-infected IL-27R-deficient mice probably through a chemokine-mediated recruitment and strategic positioning of multifunctional T cells in granulomas. As IL-27 limits optimal antimycobacterial protection by inhibiting IL-17A production, blocking of IL-27R-mediated signaling may represent a strategy for improving vaccination and host-directed therapy in tuberculosis. However, because IL-27 also prevents IL-17A-mediated immunopathology, such intervention has to be tightly controlled.
\end{abstract}

Mucosal Immunology (2018) 11:1168-1180; https://doi.org/10.1038/s41385-018-0026-3

\section{INTRODUCTION}

Mycobacterium tuberculosis (Mtb) is the causative agent of tuberculosis (TB), one of the most prevalent bacterial infections worldwide. Although TB incidence has fallen by an average of $1.5 \%$ per year since $2000,{ }^{1}$ it remains a leading global health problem. In order to develop new drug therapies and effective vaccines, it is critical to identify and understand the components and mechanisms of protective immunity against Mtb infection.

Granuloma formation in the lung is a hallmark of infection with Mtb and plays a pivotal role in host defense against mycobacteria. ${ }^{2}$ Mtb-infected macrophages in the lung initially produce various cytokines and chemokines, among them tumor necrosis factor (TNF) and the chemokines C-X-C motif ligands 9 and 10 (CXCL9 and CXCL10), which initiate the recruitment and retention of phagocytes and lymphocytes during granuloma formation. ${ }^{3-5}$ The mature lung granuloma provides a microenvironment in which antigen-specific $\mathrm{T}$ cells localize near infected macrophages and activate them for optimal bacterial killing. ${ }^{2}$ Especially $\mathrm{CD}^{+}{ }^{+} \mathrm{T}$ cells play a major role in host defense against Mtb in humans ${ }^{6,7}$ and mice. ${ }^{8,9}$ Interferon- $\gamma$ (IFN- $\gamma$ )-secreting $\mathrm{CD}^{+}{ }^{+} \mathrm{T}$ helper type 1 (Th1) cells stimulate the antimicrobial activity of infected macrophages resulting in nitric oxide synthase-2 (NOS2) production and intracellular bacterial killing. ${ }^{10}$ However, although IFN- $\gamma$ is required for protection against Mtb infection, it seems that IFN- $\gamma$ levels are not a reliable correlate of protection. ${ }^{11}$ Therefore, recent publications dealing with different infections emphasize the importance of the quality of T-cell responses in addition to their quantity. ${ }^{12,13}$ In this respect, multifunctional T cells co-expressing IFN- $\gamma$, interleukin 2 (IL-2) and TNF are superior in protection against Mtb after vaccination than singlecytokine producers, probably because they are able to secrete the highest levels of IFN- $\gamma$ and TNF on per-cell basis. ${ }^{14-16}$

We and others previously published that IL-27 is a regulator of protective $\mathrm{CD}^{+}{ }^{+}$T-cell responses and of granuloma formation during Mtb infection. ${ }^{17,18}$ IL-27, a member of the IL-12 cytokine family, is a heterodimer composed of the subunits p28 and EBI3, which are homologous to the p35 and p40 subunits of IL-12. ${ }^{19} \mathrm{IL}-27$ is mainly produced by activated dendritic cells and macrophages. ${ }^{20-22}$ It signals through a receptor consisting of a unique IL-27Ra chain and a gp130 chain shared with other IL- 6 family members. ${ }^{23}$ While IL-27 was initially reported to induce Th1 development, ${ }^{24-27}$ subsequent work revealed that this cytokine can also suppress Th1, Th17 and Th2 immune responses ${ }^{28-30}$ and support the development of IL-10-producing regulatory $\operatorname{Tr} 1$ cells. $^{3,32}$ In this context, IL-27 antagonizes IL-2 secretion by $\mathrm{T}$ cells, which is

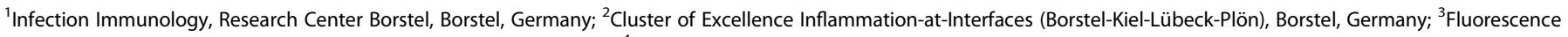
Cytometry Core Unit, Research Center Borstel, Borstel, Germany and ${ }^{4}$ Department of Infectious Disease Immunology, Statens Serum Institut, Copenhagen, Denmark Correspondence: Christoph Hölscher (choelscher@fz-borstel.de)

These authors contributed equally: Hanna Erdmann, Jochen Behrends.

Received: 9 August 2017 Revised: 27 February 2018 Accepted: 21 March 2018

Published online: 4 May 2018 
Gated on $\mathrm{CD} 90.2^{+} \mathrm{CD} 44^{+} \mathrm{CD4} 4^{+}$

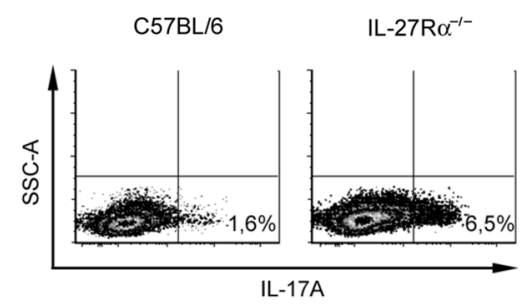

C

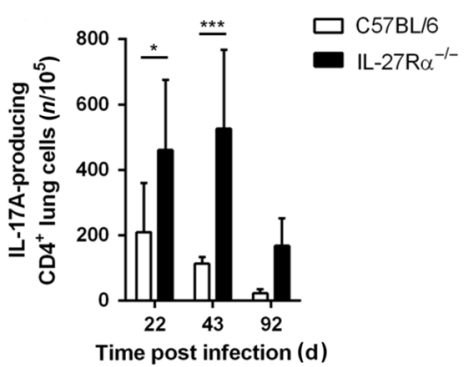

b

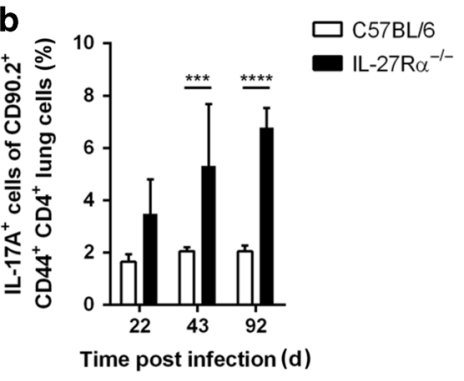

e
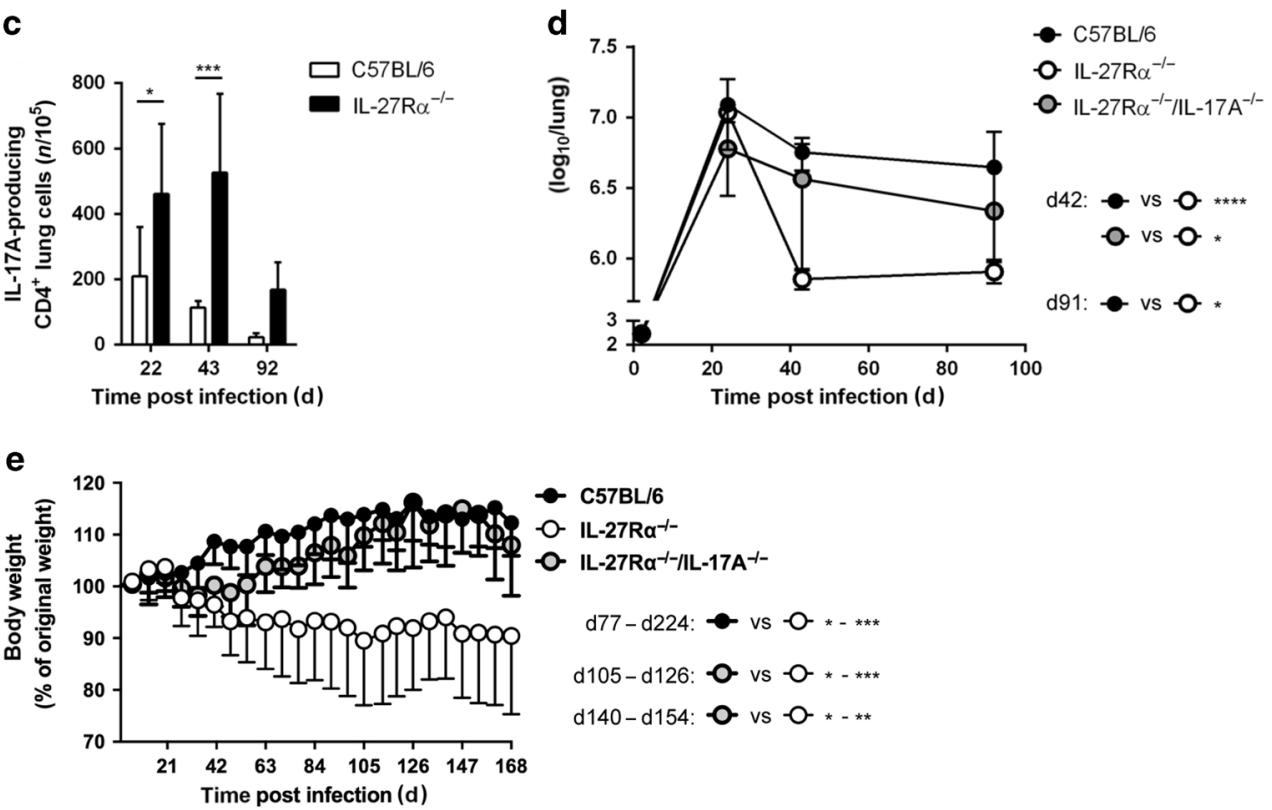

Fig. 1 The increased frequencies of IL-17A-producing $\mathrm{CD} 4^{+} \mathrm{T}$ cells in the lungs of Mtb-infected IL-27R ${ }^{-1-}$ mice are associated with an improved control of mycobacterial growth and loss of body weight. a-c C57BL/6 and IL-27R $\alpha^{-1-}$ mice were infected with $100 \mathrm{CFUs} \mathrm{H} 37 \mathrm{Rv}$ Mtb via the aerosol route. $\mathbf{a}$, b Lung cells were restimulated with immobilized anti-CD3/anti-CD28 for $4.5 \mathrm{~h}$ and analyzed for intracellular IL-17A production. a Representative density plots from day 43 post infection are shown. $\mathbf{b}$ The percentage of IL-17A-producing cells out of CD90.2 $2^{+} \mathrm{CD}_{4} 4^{+} \mathrm{CD} 4^{+}$cells over the course of infection is shown. $\mathbf{c}$ The frequencies of Esat- $6_{1-20^{-}}$specific IL-17A-producing CD4 ${ }^{+}$lung cells were determined by Elispot assay. Data represent mean \pm SD of 5 mice per group and are from one of two representative experiments performed. Statistical analysis was performed using a two-way ANOVA with Bonferroni post hoc test defining differences between C57BL/6 and IL-27R $\alpha^{-1-}$ mice as significant $\left({ }^{*} p<0.05 ;{ }^{* * *} p<0.001 ;{ }^{* * * *} p<0.0001\right)$. d, e C57BL/6, IL-27R $\alpha^{-1-}$ and IL-27R ${ }^{-1-} / \mathrm{IL}-17 \mathrm{~A}^{-1-}$ mice were infected with 100 CFUs Mtb via the aerosol route. d Mycobacterial colony enumeration assays (CFUs) in the lungs were performed. Data represent mean \pm SD of 5 mice per group and are from one of two representative experiments performed. For CFU statistics, data were log transformed and analyzed by a two-way ANOVA with Bonferroni post hoc test $\left.{ }^{*} p<0.05 ;{ }^{* * *} p<0.0001\right)$. e All animals were weighed regularly and the loss of body weight was calculated based on the initial weight. Data represent mean \pm SD of 8-10 mice per group and are from one of two representative experiments performed. Statistical analysis was performed using a two-way ANOVA with Bonferroni post hoc test defining differences between C57BL/6 and IL-27R $\alpha^{-/-}$mice as significant $\left({ }^{*} p<0.05 ;{ }^{* * *} p<0.001 ;{ }^{* * * *} p<0.0001\right)$

required for the maintenance of Th1 cells. ${ }^{33}$ Additionally, IL-27 can directly inhibit the transcription factors GATA3 ${ }^{34}$ and RORYT, thereby suppressing the development of Th2 and Th17 lymphocytes. Additionally, IL-27 and IL-12 can synergistically upregulate Blimp1 in pre-Th17 cells and polarize these to a Tr1-like subpopulation. ${ }^{36}$

Mtb infection of mice that lack the IL-27Ra chain results in lower bacterial burdens in comparison to $\mathrm{C} 57 \mathrm{BL} / 6$ wild-type mice that correlate with an increased Th1 immune response and an enhanced granuloma formation. ${ }^{17}$ Furthermore, IL-27Ra deficiency on T cells results in an altered $\mathrm{CD}^{+}$T-cell phenotype during Mtb infection, characterized by a maintained programmed cell death protein-1 (PD1), CD69 and CD127 expression and a reduced killer-cell lectin like receptor-G1 (KLRG1) expression. ${ }^{37}$ In a current model, $\mathrm{PD} 1^{+} \mathrm{CD} 4^{+}$ $T$ cells support host defense against Mtb, as they represent a pool of activated, self-renewing effector $\mathrm{T}$ cells, whereas $\mathrm{KLRG} 1^{+} \mathrm{T}$ cells exhibit a shorter life span and are not associated with protection. ${ }^{38}$ These results indicate that IL-27 is a suppressor of protective T cell- mediated immunity against Mtb in mice. IL-27 expression is also associated with active TB in humans, indicating that IL-27 inhibits protective immune responses during human TB disease. ${ }^{37}$

Because IL-27 is able to suppress the development of IL-17A-producing Th17 cells, ${ }^{29}$ we focused our work in the present study on the role of IL-17A in Mtb-infected IL-27Ra ${ }^{-1-}$ mice. IL-17A is a pro-inflammatory cytokine that is expressed by Th17 cells, but can also be produced by other cell types, like $\gamma \delta T$ cells and some innate immune cells. ${ }^{39} \mathrm{IL}-17 \mathrm{~A}$ plays a role in protection against extracellular bacterial infections by the stimulation of neutrophilrecruiting chemokines in epithelial and endothelial cells. ${ }^{40-42}$ Moreover, IL-17A is required for host resistance against intracellular pathogens by regulating adaptive immune responses or antimicrobial mechanisms in macrophages. ${ }^{3,43}$ Besides its protective functions, IL-17A is involved in the pathogenesis of several inflammatory disorders, like experimental autoimmune encephalomyelitis, psoriasis or rheumatoid arthritis. ${ }^{44-48}$ With regard to Mtb infection, IL-17A is produced during TB in mice ${ }^{49}$ and 
1170

humans ${ }^{50}$ but its role during infection is less clear. Whereas IL-17A seems not to be required for protection in mice infected with the Mtb strain $\mathrm{H} 37 \mathrm{Rv}^{49}$ it is essential for the control of the hypervirulent Mtb HN878 strain $^{51}$ by the induction of CXCL13 expression and the subsequent infiltration of $\mathrm{CXCR} 5^{+} \mathrm{T}$ cells into lung lymphoid follicles. ${ }^{51,52}$ Additionally, the induction of IL-17A secretion by vaccination correlates with protection against Mtb. ${ }^{53}$ Thus, the relevance of IL-17A for host protection seems to correlate with the induction of high levels of IL-17A production.

Because IL-27 has been shown to control IL-17A release ${ }^{29}$ we therefore hypothesized that in the absence of IL-27Ra-mediated signaling, IL-17A production is augmented during Mtb infection which eventually promotes increased protection. To proof this assumption, we compared the outcome of experimental TB in C57BL/6, IL-27Ra ${ }^{-1-}$ and IL-27Ra ${ }^{-1-}$ mice with a deficiency for IL-17A.

\section{RESULTS}

IL-17A participates in promoting the increased protection and pathology in Mtb-infected IL-27Ra ${ }^{-1-}$ mice

We have previously shown that mice deficient for IL-27Ra exhibit an increased Th1 immune response during infection with Mtb. ${ }^{17}$
Here we analyzed whether a Th17 immune response is also increased in these mice during infection. Therefore, we infected IL-27Ra ${ }^{-1-}$ mice and the corresponding C57BL/6 wild-type mice with 100 colony-forming units (CFUs) H37Rv Mtb via the aerosol route and analyzed the Th17 immune response (Fig. 1). Flow cytometric analyses revealed an increased percentage of IL-17Aproducing $\mathrm{CD}^{+} \mathrm{T}$ cells in the lungs of IL-27 $\mathrm{Ra}^{-1-}$ mice compared to C57BL/6 mice (Fig. 1a, b). By contrast, the percentage of IL-17Aproducing $\gamma \delta T$ cells was not increased upon IL-27Ra deficiency (data not shown). An ESAT6 ${ }_{1-20} 0^{-s p e c i f i c ~ E l i s p o t ~ a s s a y ~ u s i n g ~ C D 4 ~}{ }^{+}$ $T$ cells purified out of the lungs of infected animals revealed that the percentage of antigen-specific IL-17A-producing Th17 cells was also enhanced in IL-27Ra ${ }^{-1-}$ mice upon infection (Fig. 1c). As published previously, IL-27Ra ${ }^{-1-}$ mice exhibit an improved bacterial killing compared to C57BL/6 mice during Mtb infection. $^{17,18}$ To investigate whether the increased IL-17A level contributes to this protection, we next compared the outcome of Mtb infection in C57BL/6, IL-27Ra ${ }^{-1-}$ and IL-27Ra ${ }^{-1-} / \mathrm{IL}-17 \mathrm{~A}^{-1-}$ mice (Fig. 1d). Whereas the CFUs in IL-27Ra ${ }^{-1-}$ mice were significantly reduced compared to infected C57BL/6 wild-type mice, the CFUs in IL-27Ra ${ }^{-1-} / \mathrm{IL}-17 \mathrm{~A}^{-1-}$ mice were almost

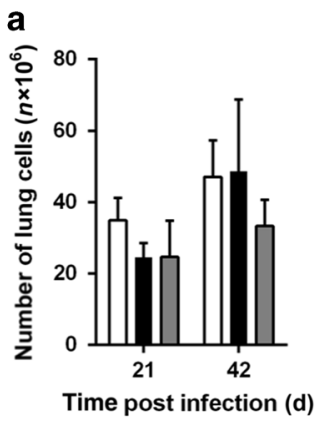

d

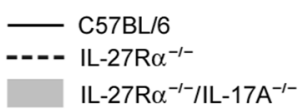

Gated on $\mathrm{CD} 90.2^{+} \mathrm{CD} 44^{+} \mathrm{CD} 4^{+}$

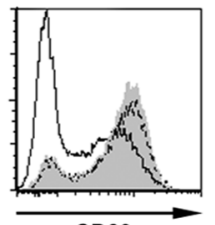

CD69

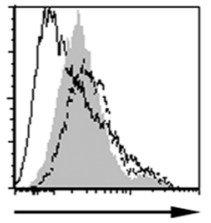

PD1 b

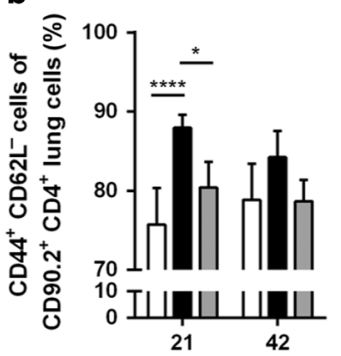

Time post infection (d)
C

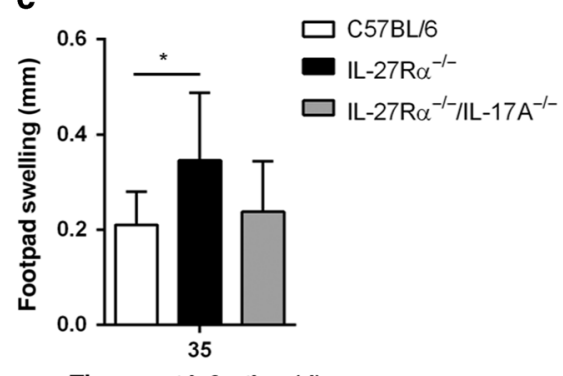

Time post infection (d)

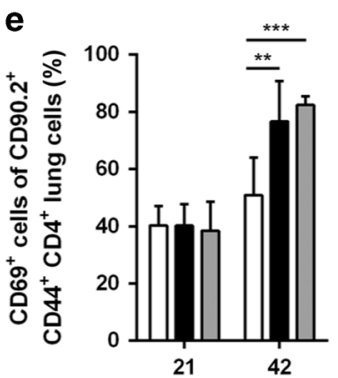

Time post infection (d)

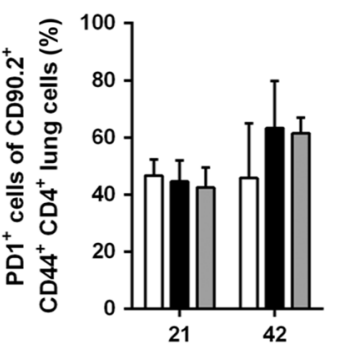

Time post infection (d)

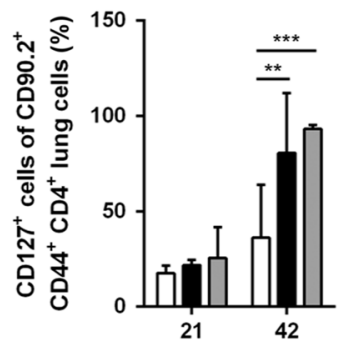

Time post infection (d)

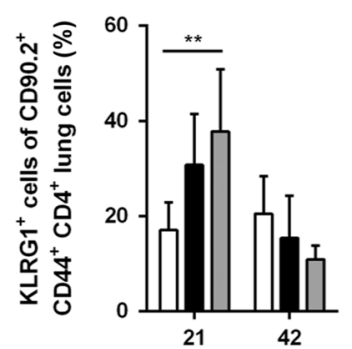

Time post infection (d)

Fig. 2 IL-17A does not affect CD69, CD127, PD1 and KLRG1 expression in the lungs of Mtb-infected IL-27R $\alpha^{-/-}$mice. C57BL/6, IL-27R $\alpha^{-/-}$and IL-27R $\alpha^{-1-} / \mathrm{IL}_{-17 \mathrm{~A}^{-/-}}$mice were infected with $100 \mathrm{CFUs}$ Mtb H37Rv via the aerosol route. a The total number of lung cells was assessed during infection. $\mathbf{b}$ The percentage of $\mathrm{CD} 44^{+} \mathrm{CD} 62 \mathrm{~L}^{-}$cells out of $\mathrm{CD} 90.2^{+} \mathrm{CD} 4^{+}$lung cells was calculated by flow cytometry. $\mathbf{c}$ The antigen-specific DTH reaction after s.c. injection of PPD was analyzed 35 days post infection. d, e Surface markers of lung cells were analyzed by flow cytometry. d Representative histograms from day 42 post infection are shown. e Percentages of CD69, CD127, PD1 and KLRG1 expressing CD90.2 ${ }^{+} \mathrm{CD}_{4}{ }^{+} \mathrm{CD} 4^{+} \mathrm{T}$ cells over the course of infection are shown. In $(\mathbf{a}, \mathbf{b}, \mathbf{d}$, e), data represent mean \pm SD of 5 mice per group. Statistical analysis was performed using a two-way ANOVA with Bonferroni post hoc test defining differences as significant $\left({ }^{*} p<0.05 ;{ }^{* *} p<0.01 ;{ }^{* * *} p<\right.$ $0.001 ; * * * *<0.0001)$. c Data represent mean \pm SD of 10-11 mice per group. Statistical analysis was performed using a one-way ANOVA with Bonferroni post hoc test defining differences as significant $\left({ }^{*} p<0.05\right)$ 
comparable to those in C57BL/6 mice. However, whereas the significant difference in bacterial loads between C57BL/6 and IL-27Ra ${ }^{-1-}$ mice was suspended in the absence of IL-17A, the CFUs in the lungs of $\mathrm{IL}-27 \mathrm{Ra}^{-1-} / \mathrm{IL}-17 \mathrm{~A}^{-1-}$ animals were-although increased-only at one time point significantly elevated compared to IL-27Ra ${ }^{-1-}$ mice. Overall, these data suggest that the increased production of IL-17A by Th17 cells supports the improved protection in the absence of the IL-27Ra. Although IL-27Ra ${ }^{-1-}$ mice are capable to better control mycobacterial growth, the increased inflammatory immune response eventually results in immunopathological consequences. ${ }^{17}$ Due to the fact that IL-17A is also strongly involved in the pathology of many chronic inflammatory and autoimmune diseases, the change of body weight was recorded after infection with $\mathrm{Mtb}$ as a mean for evaluating disease progression (Fig. 1e). During an observation period of 168 days, infected wild-type mice still gained weight, whereas Mtb infection of $\mathrm{IL}-27 \mathrm{Ra}^{-1-}$ mice resulted in a significant loss in body weight. In striking contrast, this body weight loss in IL-27Ra ${ }^{-1-}$ animals was nearly abolished when IL-17A was absent (Fig. 1e). Together, whereas the excess production of IL-17A in IL-27Ra ${ }^{-1-}$ mice contributes to increased protective immune responses against $\mathrm{Mtb}$, it also mediates the immunopathology during experimental TB.

IL-17A does not regulate CD69, CD127, PD1 and KLRG1 expression on $\mathrm{CD}^{+} \mathrm{T}$ cells in IL-27Ra ${ }^{-1-}$ mice

It is generally accepted that $\mathrm{CD}^{+} \mathrm{T}$ cells play a role in the protective immunity against Mtb. ${ }^{8,9}$ Therefore, we analyzed the phenotype of $\mathrm{CD}^{+} \mathrm{T}$ cells in C57BL/6, IL-27Ra ${ }^{-/-}$and IL-27Ra ${ }^{-1-} / \mathrm{IL}-17 \mathrm{~A}^{-/-}$mice during Mtb infection (Fig. 2). The number of total lung cells was unchanged in all three infected mouse groups (Fig. 2a). In addition, the total cell numbers of CD90.2 $2^{+}$cells and of CD90.2 $2^{+} \mathrm{CD} 4^{+} \mathrm{T}$ cells were also similar in all mice (data not shown). However, the percentage of $\mathrm{CD} 44^{+} \mathrm{CD} 2 \mathrm{~L}^{-}$cells out of $\mathrm{CD} 90.2^{+} \mathrm{CD} 4^{+}$lung cells was increased in IL-27Ra ${ }^{-1-}$ mice compared to IL-27Ra ${ }^{-1-} / \mathrm{IL}-17 \mathrm{~A}^{-1-}$ and C57BL/6 mice (Fig. 2b), indicating that IL-17A supports CD4 ${ }^{+} \mathrm{T}$ cell activation in the absence of IL-27Ra. A delayed-type hypersensitivity (DTH) reaction correlates with the degree of a T cell-mediated immune response. To monitor the development of this antigenspecific response during experimental $T B$, mice received a single subcutaneous (s.c.) injection of purified protein derivative (PPD) into the footpad at 35 days post infection, and the DTH reaction was analyzed by the measurement of the relative footpad swelling $72 \mathrm{~h}$ later (Fig. 2c). In comparison to C57BL/6 mice, IL-27Ra ${ }^{-1-}$ mice exhibited a significantly increased DTH reaction. However, the DTH in IL-27Ra ${ }^{-/}-/ \mathrm{IL}-17 \mathrm{~A}^{-/-}$mice was clearly, but not significantly, lower compared to IL-27Ra ${ }^{-1-}$ mice, suggesting a role for IL-17A in supporting antigen-specific cell-mediated immune responses to Mtb in the absence of IL-27Ra (Fig. 2c). A previous study demonstrated an impact of the IL-27R on the $\mathrm{CD}^{+}{ }^{+} \mathrm{T}$-cell phenotype, as IL-27Ra deficiency resulted in maintained PD1, CD69 and CD127 and a reduced KLRG1 expression during Mtb infection. ${ }^{37}$ We therefore investigated whether the increased IL-17A level in infected IL-27Ra ${ }^{-l-}$ mice is responsible for this altered $\mathrm{CD}^{+}{ }^{+} \mathrm{T}$-cell phenotype. In accordance with the previously published data, $C D 4^{+}$lung cells of mice that lack IL-27Ra exhibited an increased expression of CD69 and CD127 (Fig. 2d, e). However, PD1 expression and KLRG1 expression were slightly but not significantly regulated by IL-27R at 42 days post infection. Importantly, $\mathrm{CD}^{+} \mathrm{T}$ cells of IL-27Ra ${ }^{-1-} / \mathrm{IL}-17 \mathrm{~A}^{-1-}$ mice exhibited a strikingly comparable expression pattern of CD69, CD127, PD1 and KLRG1 to IL-27Ra ${ }^{-1-}$ mice (Fig. 2d, e).

This finding indicates that IL-17A does not regulate the expression of these surface markers in the absence of IL-27Ra. The fact that the increased protection of Mtb-infected IL-27Ra ${ }^{-1-}$ mice depends on IL-17A but the differential expression of CD69, CD127, PD1 and KLRG1 on $\mathrm{CD}^{+}{ }^{+} \mathrm{T}$ cells in these animals is not modulated by the lack of IL-17A points at a different IL-17A-mediated mechanism responsible for the increased protection in the IL-27Ra ${ }^{-1-}$ animals.

\section{IL-17A does not regulate $\operatorname{Tr} 1$ cell development in Mtb-infected} IL-27Ra ${ }^{-1-}$ mice

We next examined the frequency of Foxp $3^{+}$regulatory $\mathrm{T}$ cells $\left(\mathrm{T}_{\text {regs }}\right)$ and $\mathrm{IL}-10^{+}$Foxp3 $^{-}$regulatory $\mathrm{Tr} 1$ cells in Mtb-infected C57BL/6, IL $\mathrm{IL}^{-} 27 \mathrm{Ra}^{-1-}$ and IL-27Ra ${ }^{-1-} / \mathrm{IL}-17 \mathrm{~A}^{-1-}$ mice (Fig. 3). Whereas the percentage of Foxp $3^{+} \mathrm{T}_{\text {regs }}$ was similar in all three mouse groups (Fig. 3a, b), the lack of IL-27Ra resulted in a significant reduction in the frequency of Tr1 cells (Fig. 3a, c), which is in accordance with previously published data that IL-27 induces regulatory $\operatorname{Tr} 1$ cells. ${ }^{31,32}$ However, IL-27Ra ${ }^{-/-} / \mathrm{IL}-17 \mathrm{~A}^{-/-}$mice revealed a similar reduced percentage of $\operatorname{Tr} 1$ cells in the lungs of IL-27Ra ${ }^{-1-}$ mice (Fig. 3a, c), suggesting that the increased activation of $\mathrm{T}$ cells and the enhanced control of bacterial growth in IL-27Ra ${ }^{-1-}$ mice cannot be entirely ascribed to a reduction of Tr1 cells. Moreover, these data indicate that IL-17A does not regulate $\operatorname{Tr} 1$ development in the absence of IL-27Ra.

IL-17A partially regulates the expansion of multifunctional T cells in Mtb-infected IL-27Ra ${ }^{-/-}$mice

Although IFN- $\gamma$-producing $\mathrm{T}$ cells are required for protective immunity against $M t b$, the IFN- $\gamma$ level alone does not reliably correlate with protection. ${ }^{11}$ Accordingly, an ESAT6 ${ }_{1-20}$-specific Elispot assay revealed that the number of antigen-specific IFN- $\gamma$ -

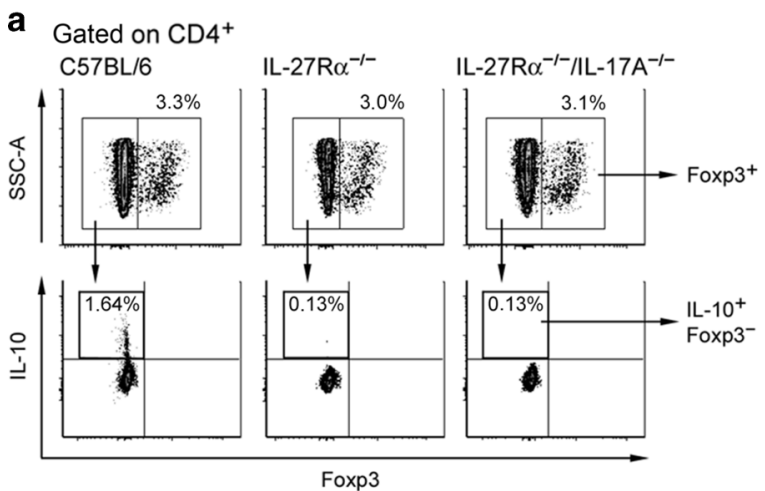

b

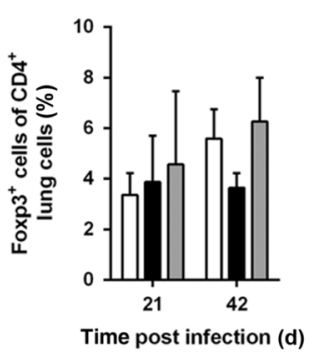

C57BL/6 c

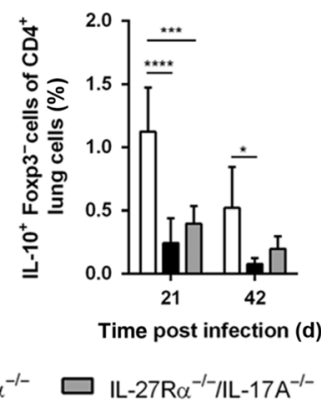

Fig. $3 \mathrm{IL}-17 \mathrm{~A}$ does not control the percentage of $\operatorname{Tr} 1$ cells in the lungs of Mtb-infected IL-27R ${ }^{-1-} \mathrm{mice}^{\mathrm{C}} \mathrm{C57BL} / 6, \mathrm{IL}-27 \mathrm{R} \alpha^{-/-}$and IL-27R $\alpha^{-/}-/ \mathrm{IL}-17 \mathrm{~A}^{-1-}$ mice were infected with $100 \mathrm{CFUs}$ Mtb H37Rv via the aerosol route. Lung cells were restimulated with immobilized antiCD3/anti-CD28 for $4.5 \mathrm{~h}$ and analyzed for intracellular Foxp3 and IL-10 production. a Representative density plots from day 21 post infection are shown. b, c Percentages of Foxp ${ }^{+}$(b) and IL- $10^{+}$Foxp3 ${ }^{-}$cells (c) of CD4 ${ }^{+}$T cells over the course of infection are shown. Data represent mean \pm SD of 5 mice per group. Statistical analysis was performed using a two-way ANOVA with Bonferroni post hoc test defining differences as significant $\left({ }^{*} p<0.05 ;{ }^{* * *} p<0.001 ;{ }^{* * * *} p<0.0001\right)$ 


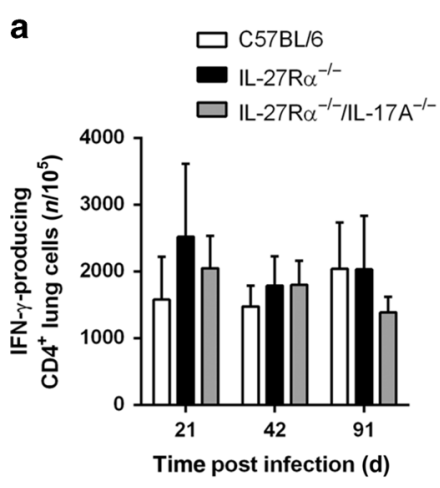

b Gated on $\mathrm{CD} 90.2^{+} \mathrm{CD} 44^{+} \mathrm{CD} 4^{+}$

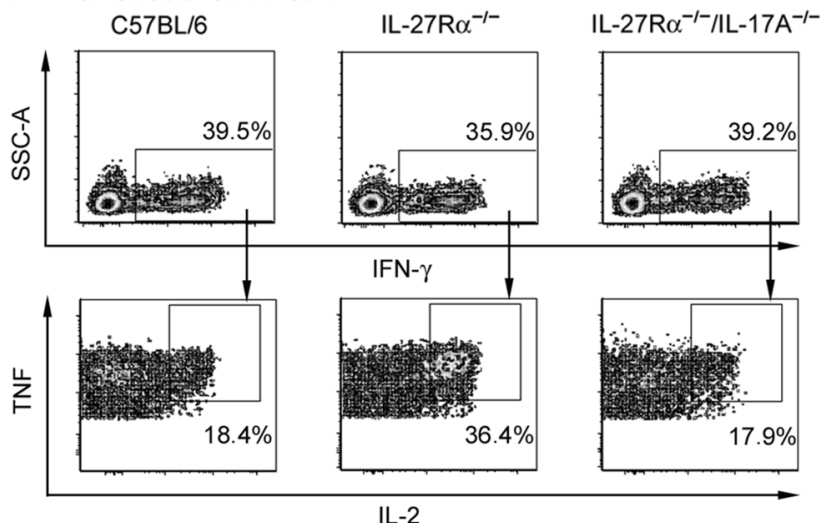

d

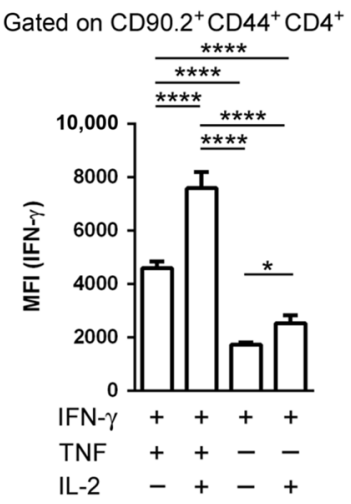

e

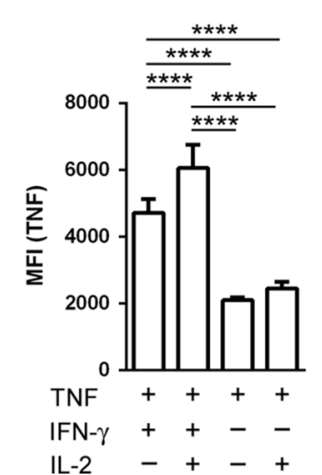

Fig. 4 IL-17A regulates the accumulation of multifunctional T cells in the lungs of Mtb-infected IL-27R $\alpha^{-1-}$ mice. C57BL/6, IL-27R $\alpha^{-/-}$and IL-27Ro ${ }^{-1-} / \mathrm{IL}_{-17 \mathrm{~A}^{-1-}}$ mice were infected with $100 \mathrm{CFUs}$ Mtb H37Rv via the aerosol route. a The frequencies of Esat- $6_{1-20^{-}}$specific IFN- $\gamma-^{-}$ producing $\mathrm{CD}^{+}{ }^{+}$lung cells were determined by Elispot assay. b-e Lung cells were restimulated with immobilized anti-CD3/anti-CD28 for $4.5 \mathrm{~h}$ and analyzed for intracellular cytokine production. $\mathbf{b}$ Representative density plots of IFN- $\gamma$-, TNF- and IL-2-co-producing multifunctional T cells on day 92 post infection are shown. c Percentages of IFN- $\gamma$-, TNF- and IL-2-producing multifunctional T cells out of CD90.2 ${ }^{+}$CD4 ${ }^{+}$CD44 ${ }^{+}$ T cells are shown. d, e CD90.2 ${ }^{+} \mathrm{CD} 44^{+} \mathrm{CD} 4^{+}$T-cell populations were divided into various cytokine-producing subsets. $\mathbf{d}$ The geometric MFI of IFN- $\gamma$ was analyzed in single-positive (IFN- $\gamma^{+}$), double-positive $\left(\mathrm{IFN}-\gamma^{+} \mathrm{TNF}^{+}\right.$and IFN $\left.\gamma^{+} \mathrm{IL}-2^{+}\right)$and triple-positive $\left(\mathrm{IFN}-\gamma^{+} \mathrm{TNF}^{+}\right.$IL-2 $\left.2^{+}\right) \mathrm{T}$ cells of C57BL/ 6 mice at day 21 post infection. e The geometric MFI of TNF was analyzed in single-positive (TNF ${ }^{+}$), double-positive $\left(\mathrm{TNF}^{+}\right.$IFN- $^{+}$and $\mathrm{TNF}^{+} \mathrm{IL}-2^{+}$) and triple-positive $\left(\mathrm{TNF}^{+} \mathrm{IFN}-\gamma^{+} \mathrm{IL}_{-2}^{+}\right.$) T cells of C57BL/6 mice at 21 days post infection. Data represent mean \pm SD of 5 mice per group and are from one of two representative experiments performed. Statistical analysis was performed using a two-way ANOVA with Bonferroni post hoc test $(\mathbf{a}, \mathbf{c})$ or a one-way ANOVA with Bonferroni post hoc test $(\mathbf{d}, \mathbf{e})$ defining differences as significant $\left({ }^{*} p<0.05\right.$; ${ }^{* * *} p<$ $0.001 ; * * * * 0<0.0001)$

producing $\mathrm{CD}^{+} \mathrm{T}$ cells in the lungs was similar in Mtb-infected C57BL/6, IL-27Ra ${ }^{-1-}$ and IL-27Ra ${ }^{-1-} / \mathrm{IL}-17 \mathrm{~A}^{-1-}$ mice (Fig. 4a). Additionally, flow cytometric analyses also revealed that the frequency of IFN- $\gamma$-producing $\mathrm{CD}^{+}{ }^{+} \mathrm{T}$ cells in the lungs was comparable in all three mouse groups (Fig. 4b). In striking contrast however, the percentage of IFN- $\gamma$-producing $\mathrm{CD}^{+}{ }^{+} \mathrm{T}$ cells that co-express TNF and IL-2 after polyclonal restimulation was elevated in IL-27Ra ${ }^{-1-}$ mice in comparison to $\mathrm{C} 57 \mathrm{BL} / 6$ and IL-27Ra ${ }^{-/-} / \mathrm{IL}-17 \mathrm{~A}^{-1-}$ mice (Fig. 4b). In fact, the frequency of these IFN- $\gamma^{-}$, TNF- and IL-2-triple-producing cells out of the CD90.2 $2^{+}$ $\mathrm{CD}_{4}{ }^{+} \mathrm{CD}^{+}$population in the lung significantly increased to approximately $12 \%$ upon IL-27Ra deficiency (Fig. 4c). Importantly, when IL-17A was absent in Mtb-infected IL-27Ra ${ }^{-1-}$ mice the relative amount of these cells dropped to a frequency of approximately $5 \%$ that was comparable to wild-type mice, suggesting that $\mathrm{IL}-17 \mathrm{~A}$ regulates the presence of polyclonal multifunctional $\mathrm{CD}^{+} \mathrm{T}$ cells. Hence, the IL-17A-dependent expansion of these multifunctional $\mathrm{T}$ cells may contribute to the increased protection of IL-27Ra ${ }^{-1-}$ mice after Mtb infection. In line with this perception, the IFN- $\gamma$-, TNF- and IL-2-co-producing multifunctional $T$ cells are also the most efficient producers of these cytokines on a per-cell basis because when we measured the relative concentrations of IFN- $\gamma$ and TNF as the geometric mean fluorescence intensities (MFIs) in various cytokine-producing $\mathrm{CD}^{+} \mathrm{T}$ cells of infected C57BL/6 mice, the highest MFIs for IFN- $\gamma$ and TNF were found in triple-positive T cells (Fig. 4d, e). However, the increased MFI of these cytokines in multifunctional T cells was not further enhanced in IL-27Ra ${ }^{-/-}$and IL-27Ra ${ }^{-1-} / \mathrm{IL}-17 \mathrm{~A}^{-1-}$ mice (data not shown).

To further delineate the antigen specificity of these multifunctional $T$ cells, we first determined the frequency of antigenspecific activated CD4 T cells by co-staining of lung cells of infected mice with $\mathrm{CD} 90.2^{+} \mathrm{CD}_{4}{ }^{+} \mathrm{CD}^{+}$and $\mathrm{IA}_{\mathrm{b}} \mathrm{ESAT6}_{4-17}$ tetramers (Fig. 5a). Flow cytometric analysis revealed that the frequencies of antigen-specific cells in the CD90.2 ${ }^{+}$ $\mathrm{CD}_{4}{ }^{+} \mathrm{CD}^{+}$population were similar in $\mathrm{C} 57 \mathrm{BL} / 6, \mathrm{IL}-27 \mathrm{Ra}^{-1-}$ and IL-27Ra ${ }^{-1-} / \mathrm{IL}-17 \mathrm{~A}^{-1-}$ mice (Fig. 5b). The percentage of IFN- $\gamma-$ producing $\mathrm{CD}^{+}{ }^{+} \mathrm{T}$ cells that co-express TNF and IL-2 after antigenspecific restimulation with short-term culture filtrate (ST-CF) was approximately $0.25 \%$ similar in all three mouse strains after 35 days of infection (Fig. 5c). After 48 days, the proportion of antigen-specific multifunctional T cells was not changed in wildtype mice but significantly increased to $0.75 \%$ in IL-27Ra ${ }^{-1-}$ mice. However, in the absence of IL-17A the relative amount of these cells was only partially reduced in Mtb-infected IL-27Ra ${ }^{-/-}$mice (Fig. 5 C). So far, our data suggest that IL-27 not only promotes the 
a Gated on $\mathrm{CD} 90.2^{+} \mathrm{CD}_{4} 4^{+} \mathrm{CD} 4^{+}$
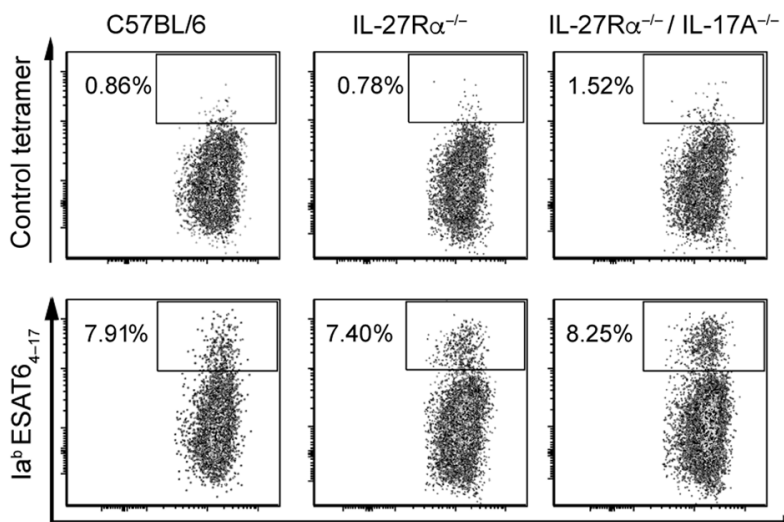

CD4
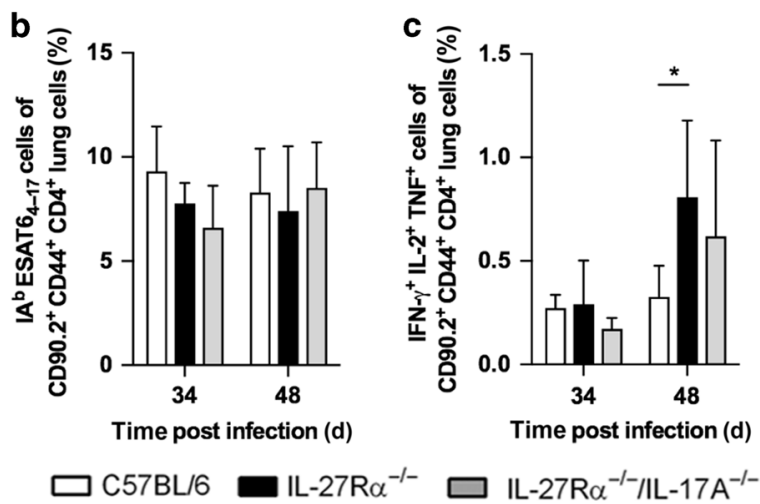

Fig. 5 Increased proportion of multifunctional T cells in the antigenspecific CD4 T-cell population in the lungs of Mtb-infected IL-27R $\alpha^{-1}$ mice. C57BL/6, IL-27R $\alpha^{-1-}$ and IL-27R $\alpha^{-1-} / \mathrm{IL}-17 \mathrm{~A}^{-1-}$ mice were infected with 100 CFUs Mtb H37Rv via the aerosol route. a, b The frequencies of $\mathrm{IA}^{\mathrm{b}} \mathrm{ESAT6}_{4-17^{-}}$specific CD90.2 ${ }^{+} \mathrm{CD} 4^{+} \mathrm{CD}^{+} 4^{+}$lung cells were determined by flow cytometry. a Representative density plots of IA $^{\text {b ESAT6 }}{ }_{4-17}$-positive out of CD90.2 ${ }^{+} \mathrm{CD4}^{+} \mathrm{CD} 4^{+} \mathrm{T}$ cells on day 48 post infection. The upper panel shows staining with control tetramers. b Percentages of IA ${ }^{\mathrm{E}} \mathrm{ESAT6}_{4-17}$-positive out of $\mathrm{CD} 90.2^{+} \mathrm{CD}^{+} \mathrm{CD}^{+} 4^{+}$ T cells cells are shown. c Lung cells were restimulated with ST-CF for $4.5 \mathrm{~h}$ and analyzed for intracellular cytokine production. Percentages of IFN- $\gamma$-, TNF- and IL-2-producing multifunctional T cells out of CD90.2 ${ }^{+} \mathrm{CD}^{+} \mathrm{CD}^{+} 4^{+} \mathrm{T}$ cells are shown. Data represent mean $\pm \mathrm{SD}$ of 5 mice per group. Statistical analysis was performed using a two-way ANOVA with Bonferroni post hoc test defining differences as significant $\left({ }^{*} p<0.05\right)$

expansion of polyclonal IFN- $\gamma$-, TNF- and IL-2-triple-producing CD4 $\mathrm{T}$ cells, but also clearly mediates the development of antigenspecific multifunctional T cells. However, whereas the increase of polyclonal multifunctional $T$ cells in the absence of IL-27R is eventually significantly dependent on IL-17A, the expansion of antigen-specific IFN- $\gamma-$, TNF- and IL-2-triple-producing CD4 T cells appears to be only partially mediated by IL-17A.

IL-17A seems not to be required for protection in mice infected with the Mtb strain $\mathrm{H} 37 \mathrm{Rv}^{49}$ (that we have used so far in the present study) but it is essential for the control of the hypervirulent Mtb HN878 strain. ${ }^{51}$ Therefore, we further speculated that this IL-17A-dependent protection after infection with Mtb HN878 is also associated with the accumulation of multifunction CD4 T cells and compared the frequencies of IFN- $\gamma^{-}$, TNFand IL-2-triple-producing CD4 T cells in wild-type and IL-17A ${ }^{-1-}$ mice after infection with Mtb H37Rv and Mtb HN878 (Fig. 6). After 21 and 42 days of infection with Mtb H37Rv, no differences in the frequencies of multifunctional cells in the CD90.2 $2^{+} \mathrm{CD} 44^{+} \mathrm{CD} 4^{+}$ population were observed between wild-type and IL-17 ${ }^{-/-}$mice after restimulation with anti-CD3/CD28 (Fig. 6a, left). In contrast, at 49 days after infection with Mtb HN878, the expansion of polyclonal multifunctional CD4 T cells was significantly reduced in the lungs of IL-17A $\mathrm{A}^{-1-}$ animals (Fig. $6 \mathrm{a}$, right). However, when lung cells of mice that were infected with Mtb HN878 were restimulated with ST-CF, the frequency of IFN- $\gamma^{-}$, TNF- and IL-2-triple-producing CD4 T cells in IL-17A ${ }^{-1-}$ was only slightly reduced on day 49 (Fig. 6b). By comparing the expansion of multifunctional cells in the CD90.2 $2^{+} \mathrm{CD} 44^{+} \mathrm{CD} 4^{+}$population in the lungs of wild-type and IL-17A ${ }^{-1-}$ mice after infection with Mtb H37Rv and the highly virulent strain Mtb HN878, we give evidence that the accumulation of multifunctional $T$ cells is partially mediated by high levels of IL-17A.

During experimental TB, IL-27R regulates gene expression of $\mathrm{CCl} 20$ and the accumulation of CCR6-expressing CD4 T cells

C-C chemokine receptor 6 (CCR6) is expressed by Th17 cells ${ }^{54,55}$ and is critical for the migration of murine Th17 cells into inflamed tissues. ${ }^{56,57}$ Therefore, we were wondering whether during experimental TB the frequency of antigen-specific CCR6-expressing CD4 T cells is modulated in the absence of IL-27R-mediated signaling (Fig. 7a). At both examined time points after infection, the relative amount of Mtb-specific CCR6-positive activated CD4 T cells was significantly increased in the lungs of IL-27Ra ${ }^{-1-}$ mice when compared to the frequencies of these cells isolated from wild-type mice (Fig. 7b). Importantly, IL-17A-deficiency in IL-27Ra ${ }^{-1-}$ mice had no effect on this Mtb-specific CCR6-positive CD4 T-cell population. CCL20, the ligand for CCR6, is expressed by various cell types, among them lung epithelial cells ${ }^{58}$ and also Th17 cells. ${ }^{54}$ Because IL-27 appears to hinder the accumulation of CCR6-expressing CD4 T cells, we speculated whether this effect was accompanied by a modulated CCL20 expression. In fact, during the course of experimental TB, CCl20 gene expression in the lungs of IL-27Ra ${ }^{-1-}$ mice was significantly enhanced when compared to transcript levels in wild-type mice (Fig. 7c). Importantly, the absence of IL-17A initially further enhanced CCl20 gene expression in IL-27Ra ${ }^{-/-}$mice, but at later time points CCl20 was similarly expressed in single- and double-deficient animals. Together, our data give evidence that in experimental TB, IL-27 modulates the recruitment of CCR6 CD4 T cells indirectly and independent of IL-17A by inhibiting the expression of CCL20.

IL-17A stimulates $\mathrm{Cxcl9}, \mathrm{Cxcl} 10$ and $\mathrm{Cxcl} 13$ chemokine expression in IL-27Ra ${ }^{-1-}$ mice after Mtb infection

Previous data suggest that IL-17A induces CXCL9 and CXCL10 to recruit protective IFN- $\gamma$-producing Th1 memory cells after subunit vaccination against Mtb. ${ }^{53}$ Additionally, an IL-17A-induced CXCL13 expression appears to be important for the localization of $\mathrm{CXCR5}^{+}$ $T$ cells in inducible bronchus-associated lymphoid tissue (iBALT) of lung granulomas. ${ }^{51,52}$ Therefore, we examined the expression of these chemokines in lung homogenates of Mtb-infected C57BL/6, IL-27Ra ${ }^{-1-}$ and IL-27Ra ${ }^{-1-} / \mathrm{IL}-17 \mathrm{~A}^{-1-}$ mice by quantitative real-time PCR. The increased IL-17A production in Mtb-infected IL-27Ra ${ }^{-/-}$ mice was associated with an enhanced $\mathrm{CxCl9}, \mathrm{CxCl} 10$ and $\mathrm{CxCl} 13$ chemokine expression in the lungs, whereas the expression of these chemokines was comparable after infection in IL-27Ra ${ }^{-1-}$ mice to that in C57BL/6 mice when IL-17A was absent (Fig. 8). As IL-17A-induced Cxcl13 expression supports the localization of T cells within lung lymphoid follicles, ${ }^{51,52}$ we next analyzed the granulomatous response in the three infected mouse groups.

IL-17A supports granuloma formation in the lungs of Mtb-infected IL-27Ra ${ }^{-1-}$ mice

We previously published that IL-27Ra ${ }^{-/-}$mice exhibit an effective granulomatous response in the lung tissue upon Mtb infection. ${ }^{17}$ Since IL-17A appears to be important for granuloma formation during experimental TB, ${ }^{51,59}$ we next analyzed the role of IL-17A in 
a

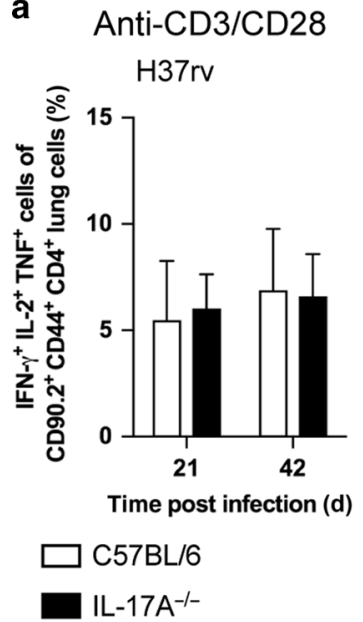

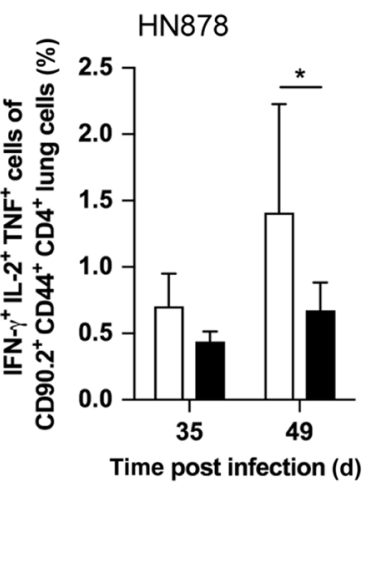

b $\mathrm{ST}-\mathrm{CF}$

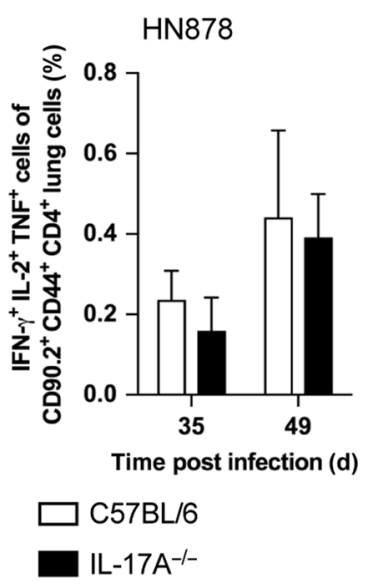

Fig. 6 Infection with the highly virulent Mtb strain HN878 results in increased frequencies of polyclonal multifunctional T cells even in IL$17 \mathrm{~A}^{-1-}$ mice. C57BL/6 and IL-17A ${ }^{-1-}$ mice were infected with 100 CFUs Mtb H37Rv or HN878 via the aerosol route. Lung cells were restimulated with a immobilized anti-CD3/anti-CD28 or b ST-CF for $4.5 \mathrm{~h}$ and analyzed for intracellular cytokine production. Percentages of IFN- $\gamma-$, TNF- and IL-2-producing multifunctional T cells out of CD90.2 ${ }^{+} \mathrm{CD} 4^{+} \mathrm{CD} 44^{+}$T cells are shown. Data represent mean \pm SD of 5 mice per group. Statistical analysis was performed using a two-way ANOVA with Bonferroni post hoc test defining differences as significant $\left({ }^{*} p<0.05\right)$

lung granuloma formation of Mtb-infected IL-27Ra ${ }^{-1-}$ mice (Fig. 9). In comparison to $\mathrm{C} 57 \mathrm{BL} / 6$ mice, which exhibited rather unstructured granulomas during infection, granulomas in the lungs of IL-27Ra ${ }^{-1-}$ mice were found to be highly stratified (Fig. 9a) with a rim of T (Fig. 9b) and B cells (Fig. 9c) and a center of Mtbcontaining macrophages. Importantly, the increased level of IL-17A was responsible for this efficient granulomatous response in IL-27Ra ${ }^{-1-}$ mice, because granulomas in IL-27Ra ${ }^{-1-} / \mathrm{IL}-17 \mathrm{~A}^{-1-}$ mice were unstructured again and similar to those in C57BL/6 mice (Fig. 9a-c). We next suggested that the IL-17A-induced strategic positioning of immune cells in the highly stratified granulomas of $\mathrm{IL}-27 \mathrm{Ra}^{-1-}$ mice eventually results in a better activation of macrophages and a more efficient elimination of Mtb. Therefore, we analyzed Nos2 expression in lung homogenates by quantitative real-time PCR (Fig. 9d) and the protein expression in histological sections (Fig. 9e). The increased IL-17A expression in IL-27Ra ${ }^{-/-}$mice correlated with an increased NOS2 expression in the lung (Fig. 9d). Importantly, NOS2-expressing macrophages were found to be located mainly in the center of the structured granulomas of Mtb-infected IL-27Ra ${ }^{-1-}$ mice (Fig. 9e), suggesting that the IL-17A-induced strategic positioning of immune cells leads to an optimal macrophage activation and elimination of Mtb in the absence of IL-27Ra-mediated signaling.

\section{DISCUSSION}

IL-27 is an IL-12 cytokine family member with anti-inflammatory properties. It has broad inhibitory effects on Th1, Th2 and Th17 immune responses ${ }^{28-30,60}$ and induces the development of regulatory IL-10-secreting Tr1 cells. ${ }^{31,32}$ In humans, IL-27 expression is associated with active TB, indicating that IL-27 inhibits protective immunity during human TB. ${ }^{37}$

With regard to experimental Mtb infection it has previously been shown that IL-27 inhibits protective immune responses leading to an impaired control of Mtb in mice. ${ }^{17,18}$ Thus, IL-27Ra ${ }^{-/-}$mice exhibit significantly decreased bacterial loads during Mtb infection compared to C57BL/6 mice, which is associated with a higher production of the pro-inflammatory cytokines IL-12 and TNF, an elevated T-cell activation and enhanced macrophage effector functions. ${ }^{17}$ In the present study we show for the first time that the IL-17A production by Th17 cells is also significantly increased in Mtb-infected IL-27Ra ${ }^{-1-}$ mice. We therefore further analyzed a potential role of IL-17A for protection against Mtb in the absence of IL-27Ra-mediated signaling.

Lung-resident $\gamma \delta T$ cells are the first "innate" producers of IL-17A during experimental Mtb infection. ${ }^{61}$ However, when the adaptive immune response develops, antigen-specific Th17 cells are induced that secrete IL-17A. ${ }^{49,53}$ We observed that IL-27Ra ${ }^{-1-}$ mice exhibit a significant increase of IL-17A-producing $\mathrm{CD}^{+}$ $T$ cells during Mtb infection, but the frequency of IL-17Aproducing $\gamma \delta T$ cells was not enhanced (data not shown), suggesting that IL-27 specifically targets IL-17A-producing Th17 cells. In this respect, we here give evidence that the accumulation of CCR6-expressing Th17 cells is controlled by IL-27 through the inhibition of CCL20 expression. CCL20, the ligand for CCR6, is expressed by different types of cells but particularly by lung epithelial cells. ${ }^{58}$ Although we have not identified the cellular source of CCL20 in Mtb-infected IL-27Ra ${ }^{-/-}$mice, we could not exclude that IL-27 modulates the recruitment of Th17 cells indirectly by inhibiting the expression of CCL20, e.g., in lung epithelial cells.

By comparing the outcome of Mtb infection in C57BL/6, IL-27Ra ${ }^{-1-}$ and IL-27Ra ${ }^{-1-} / \mathrm{IL}-17 \mathrm{~A}^{-/-}$mice we demonstrate that the increased protection of IL-27Ra ${ }^{-1-}$ animals can be ascribed in part to the augmented IL-17A secretion. In general, the relevance of IL-17A for host protection against Mtb seems to correlate with the induction of high levels of IL-17A. Thus, a low-dose infection of $\mathrm{IL}-17 \mathrm{~A}^{-1-}$ mice with the lab-adapted strain Mtb H37Rv does not result in increased bacterial load in comparison to C57BL/6 mice. ${ }^{52}$ Also, in low-dose Mtb-infected IL-23p19 ${ }^{-1-}$ mice, which exhibit a decreased Th17 response, bacterial loads were unaltered when compared to wild-type mice. ${ }^{49}$ This suggests that in C57BL/6 mice IL-17A is induced suboptimally by Mtb H37Rv and that IL-17A is not required for early control of this bacterial strain. However, the boosted induction of an IL-17A secretion by vaccination ${ }^{53}$ or by the infection of mice with more virulent Mtb strains ${ }^{52}$ correlates with protection. Furthermore, it was possible to improve the control of Mtb infection when IL-23, which is required to sustain Th17 responses and IL-17A messenger RNA (mRNA) expression in experimental $\mathrm{TB}^{49}$ was delivered prior to infection. ${ }^{62}$ Therefore, we hypothesize that the significant increase in IL-17A production in Mtb-infected $\mathrm{IL}_{-27 \mathrm{Ra}^{-1-}}$ mice also promotes protective immune responses. In C57/BL/6 mice, this protective IL-17Amediated immune reaction is probably limited by IL-27 in order to prevent IL-17A-induced immunopathology. 


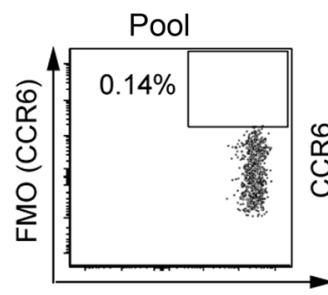

CD4

b

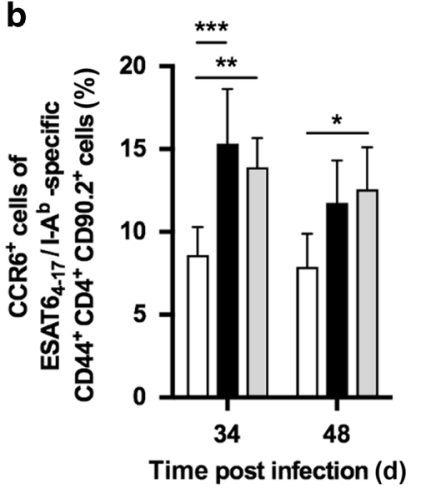

C57BL/6
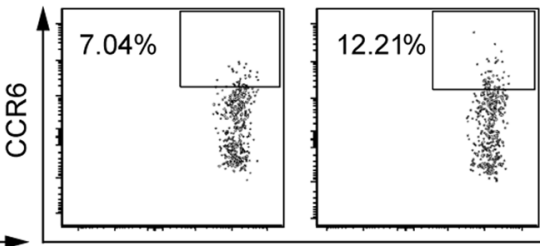

CD4
IL-27R $\alpha^{-1-} / \mathrm{IL}-17 \mathrm{~A}^{-/-}$

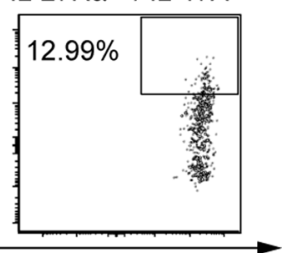

C

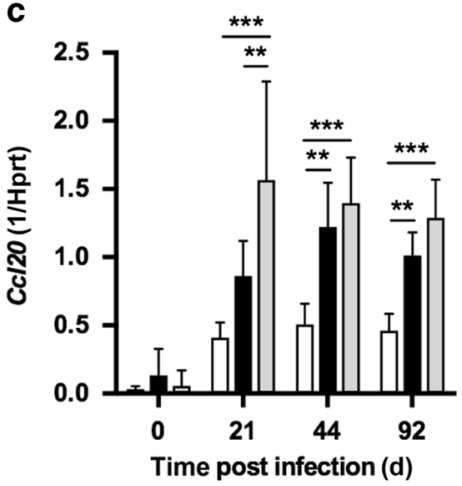

IL-27R $\alpha^{-/-}$

Fig. 7 Increased frequencies of CCR6 ${ }^{+} \mathrm{CD} 4 \mathrm{~T}$ cells and $\mathrm{Ccl} 20$ gene expression in the antigen-specific CD4 T-cell population in the lungs of Mtb-infected IL-27R $\alpha^{-1-}$ mice. C57BL/6, IL-27R $\alpha^{-1-}$ and IL-27R $\alpha^{-1-} /$ IL-17A ${ }^{-1-}$ mice were infected with 100 CFUs Mtb H37Rv via the aerosol route. $\mathbf{a}$, b The frequencies of CCR6-positive $\mathrm{IA}^{\mathrm{b}} \mathrm{ESAT6}{ }_{4-17}{ }^{+} \mathrm{CD}_{4} 4^{+} \mathrm{CD} 4^{+} \mathrm{CD} 90.2^{+}$lung cells were determined by flow cytometry. a Representative density plots of CCR6-positive IA ${ }^{\mathrm{b}} \mathrm{ESAT6}_{4-17}{ }^{+} \mathrm{CD}_{4}{ }^{+} \mathrm{CD}^{+}{ }^{+} \mathrm{CD} 90.2^{+}$cells on day 48 post infection. As control FMO (CCR6) staining of pooled cells was performed. b Percentages of CCR6-positive out of IA ${ }^{\mathrm{b}} \mathrm{ESAT6}_{4-17}{ }^{+} \mathrm{CD}_{4} 4^{+}$CD4 $4^{+}$CD90.2 ${ }^{+}$cells are shown. $\mathbf{c}$ Gene expression of $\mathrm{CCl} 20$ in lung homogenates was quantified by real-time PCR at the indicated time points post infection. Data represent mean \pm SD of 5 mice per group. Statistical analysis was performed using a two-way ANOVA with Bonferroni post hoc test defining differences as significant $\left({ }^{*} p<0.05 ;{ }^{* *} p<0.01 ;{ }^{* *} p<0.001\right)$

Previous publications suggest that during Mtb infection T cells can be divided into two populations, which express either KLRG1 or PD $1 .{ }^{38}$ Whereas $\mathrm{KLRG}^{+} \mathrm{T}$ cells are located in the lung vasculature and are irrelevant for host immunity against Mtb, $\mathrm{PD}^{+} \mathrm{T}$ cells migrate to the lung parenchyma and contribute to host resistance. ${ }^{63}$ IL-27Ra appears to regulate the phenotype of $\mathrm{T}$ cells, because IL-27Ra ${ }^{-1-}$ mice exhibit CD4 ${ }^{+} \mathrm{T}$ cells which are characterized by a maintained PD1, CD69 and CD127 and a decreased KLRG1 expression. ${ }^{37}$ Therefore, it was speculated that the increased protection in $\mathrm{L}-27 \mathrm{Ra}^{-1-}$ mice is a consequence of this altered $\mathrm{CD}^{+}{ }^{+}$-cell phenotype. In the present study, we also observed an increased CD69, CD127 and PD1 expression upon IL-27Ra deficiency. However, this $\mathrm{CD}^{+}$T-cell phenotype in infected IL-27Ra ${ }^{-1-}$ mice was not affected by the absence of IL-17A. Because to the contrary the increased protection of IL-27Ra ${ }^{-1-}$ mice against Mtb infection was lost when IL-17A was lacking, the sustained PD1, CD69 and CD127 and reduced KLRG1 expression on $\mathrm{CD}^{+} \mathrm{T}$ cells appears not to be sufficient to improve the control of Mtb. Furthermore, IL-27 is also known to regulate protective immunity by other mechanisms. Because IL-27 induces the development of IL-10-secreting $\operatorname{Tr} 1$ cells, ${ }^{31,32}$ we hypothesized that a reduction of these cells in Mtb-infected $\mathrm{IL}-27 \mathrm{Ra}^{-/-}$mice might also contribute to the increased protection of these mice. Indeed, the frequency of IL-10-producing Foxp $3^{-} \mathrm{CD}^{+}{ }^{+} \mathrm{T}$ cells was significantly reduced in IL-27Ra ${ }^{-1-}$ mice. However, infected IL-27Ra ${ }^{-1-} /$ IL-17A ${ }^{-1-}$ mice also exhibited decreased percentages of $\operatorname{Tr} 1$ cells. Hence, the decreased presence of Tr1 cells appears not to account for the improved immune response in Mtb-infected IL-27Ra ${ }^{-1-}$ mice.

We show here that an induced IL-17A secretion in the lungs of infected IL-27Ra ${ }^{-1-}$ mice correlates with increased frequencies of
IFN- $\gamma^{-}$, IL-2- and TNF-secreting multifunctional T cells. Additionally, the augmented percentage of these cells was reduced in the lungs of infected IL-27Ra ${ }^{-/} / \mathrm{IL}-17 \mathrm{~A}^{-/-}$mice, indicating that IL-17A contributes to the expansion of these cells during Mtb infection. However, whereas the accumulation of polyclonal multifunctional $\mathrm{T}$ cells was strikingly dependent on IL-17A, IL-17A appears to contribute only to a minor degree to the increase of antigenspecific cells in IL-27Ra ${ }^{-1-}$ mice. The relative low frequency and late appearance of IFN- $\gamma$-, TNF- and IL-2-triple-producing CD4 $T$ cells after antigen-specific restimulation, however, may have affected a clear distinguishability. Because infection of wild-type mice with the hypervirulent Mtb HN878 strain recapitulates the IL-17A-dependent expansion of multifunctional $T$ cells in the absence of IL-27R-mediated signaling after infection with the Mtb $\mathrm{H} 37 \mathrm{Rv}$ strain, the level of IL-17A induction appears to be essential for the accumulation of IFN- $\gamma-$, TNF- and IL-2-triple-producing CD4 T cells. ${ }^{51}$

Nevertheless, it has been previously suggested that IFN- $\gamma$ alone may be necessary but not sufficient for host resistance against Mtb. ${ }^{64}$ Multifunctional T cells co-expressing IFN- $\gamma$, IL-2 and TNF are superior in protection against Mtb after vaccination than singlecytokine producers. ${ }^{14-16}$ The protective capacity of these T cells correlate with their ability to secrete high levels of cytokines per cell after vaccination against Mtb (see refs. ${ }^{14-16}$ ) or against other pathogens, like Leishmania major ${ }^{12}$ or Plasmodium falciparum. ${ }^{65}$ Also the secretion of IFN- $\gamma$ and TNF from the same cell might have a synergistic effect on macrophage effector mechanisms against Mtb. ${ }^{15,66}$ Hence, the capability of the host to respond to Mtb infection with an efficient production of IL-17A is a prerequisite for a more effective generation of multifunctional $T$ cells and subsequent better protection. The mechanism by which IL-17A 


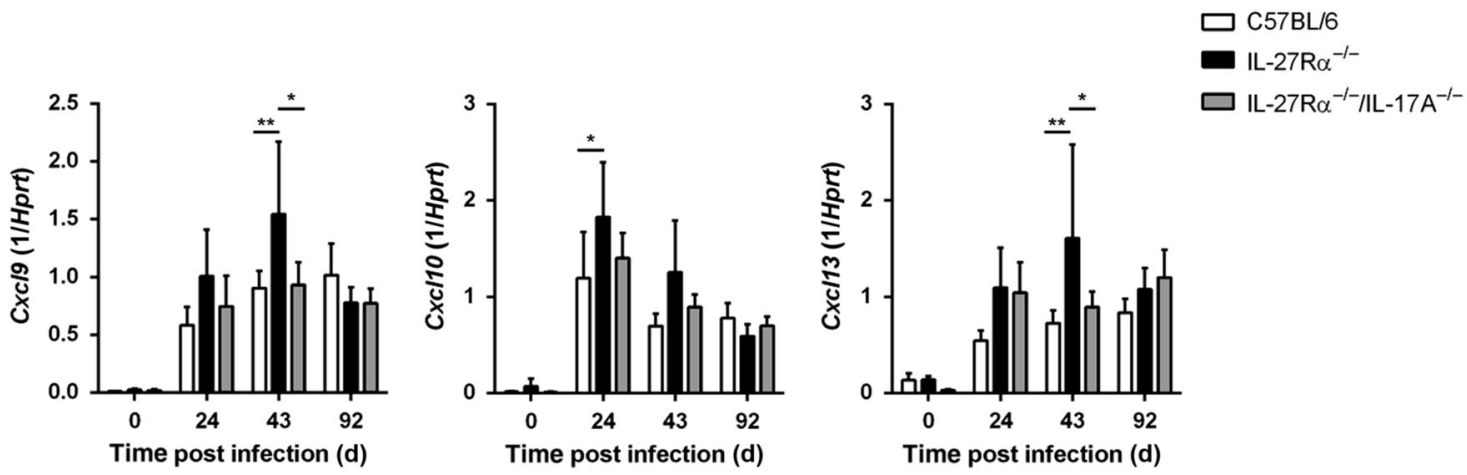

Fig. 8 IL-17A promotes CXCL chemokine expression in the lungs of Mtb-infected IL-27R $\alpha^{-1-} \mathrm{mice}^{\mathrm{C}} \mathrm{C57BL/6}, \mathrm{IL}-27 \mathrm{R} \alpha^{-1-}$ and IL-27R $\alpha^{-/-} /$IL-17A $\mathrm{A}^{-1-}$ mice were infected with 100 CFUs Mtb H37Rv via the aerosol route. The gene expression of $C x C / 9, C x c / 10$ and CxCl13 in lung homogenates was quantified by real-time PCR at the indicated time points post infection. Data represent mean \pm SD of 5 mice per group and are from one of two representative experiments performed. Statistical analysis was performed using a two-way ANOVA with Bonferroni post hoc test defining differences as significant $\left({ }^{*} p<0.05 ;{ }^{* *} p<0.01\right)$

may promote the accumulation of multifunctional $T$ cells in the lung is unclear. However, we also observed a partially increased IL-17A-dependent expression of CXCL9 and CXCL10 in the lungs of IL-27Ra ${ }^{-1-}$ mice, which might contribute to the recruitment of these protective T cells. Accordingly, in subunit-vaccinated mice, the expression of IL-17A mRNA correlated with the expression of the chemokines CXCL9, CXCL10 and CXCL11 and the induction of an accelerated Th1 response, which was reduced after IL-17A depletion. ${ }^{53}$ Therefore, we suggest that IL-17A contributes to the increased protection in the absence of IL-27Ra by the chemokinemediated accumulation of protective multifunctional $\mathrm{T}$ cells-a function that has not been ascribed to IL-17A before.

For optimal Mtb control, T cells need to co-localize with infected macrophages in order to activate anti-mycobacterial effector mechanisms. Hence, granuloma formation in the lung is a hallmark of Mtb infection and plays a pivotal role in host defense against mycobacteria. ${ }^{2}$ Accordingly, highly stratified formation of structured granulomas is a prerequisite for an optimal immune defense. We observed in the lungs of Mtb-infected IL-27Ra ${ }^{-1-}$ mice the development of extremely well-organized granulomas with a core of activated macrophages surrounded by a rim of lymphocytes which was clearly dependent on IL-17A. Therefore, we conclude that IL-17A supports the strategic positioning of protective $\mathrm{T}$ cells around infected macrophages and thereby contributes to the improved protection in the absence of IL-27Ramediated signaling. Previous reports already suggest a role for IL-17A in granuloma formation during experimental TB. After highdose intratracheal infection with $\mathrm{Mtb}, \mathrm{IL}-17 \mathrm{~A}^{-1-}$ mice exhibited a decreased protection against bacteria, which was associated with an impaired granuloma formation in the lung. ${ }^{59}$ Additionally, Mtbinfected IL-23p $19^{-1-}$ mice exhibit very little IL-17A mRNA production in the lung and this also correlates with an altered granulomatous response. ${ }^{49}$ Furthermore, it was demonstrated that IL-17A-induced CXCL13 is important for the correct migration of $\mathrm{CXCR5}^{+} \mathrm{T}$ cells within the granuloma. ${ }^{51,52,67}$ Along this line, IL-17A is also required for the CXCL13-dependent formation of iBALT, $^{68}$ which appears to be critical in TB for the eutopic generation and proliferation of antigen-specific $T$ cells in the lung. ${ }^{69}$ We also observed a partial IL-17A-dependent increased expression of $\mathrm{CxCl} / 3$ in IL-27Ra ${ }^{-1-}$ mice, suggesting that the enhanced IL-17A production in the absence of IL-27Ra-mediated signaling contributes to the formation of highly stratified protective granulomas by inducing CXCL13 expression. Also, B cells accumulate in secondary lymphoid structures in mice and humans during TB. ${ }^{70-72}$ After experimental infection with Mtb, B cell-deficient mice exhibit an altered granulomatous response ${ }^{73}$ accompanied by reduced protection. ${ }^{74}$ Our observations also demonstrate that a large component of the lymphocytic rim of the granulomas in IL-27Ra ${ }^{-1-}$ mice consists of B cells. Therefore, the IL-17A-dependent localization of B cells, presumably also by the induction of CXCL13, might also contribute to the improved protection against Mtb.

Furthermore, IL-17A secretion is often associated with neutrophil recruitment, which in turn is associated with early granuloma formation after Mtb infection. ${ }^{75}$ We observed by flow cytometry that Mtb-infected IL-27Ra ${ }^{-1-}$ mice also exhibited an IL-17A-dependent early accumulation of neutrophils in their lungs and microscopical analyses revealed that these neutrophils accumulate in the centers of the structured granulomas (data not shown). However, albeit treatment of IL-27Ra ${ }^{-1-}$ mice with anti-G-CSF during the first 37 days post Mtb infection leads to a reduction of neutrophils in the blood, the granuloma formation, chemokine expression and bacterial burdens were not affected (data not shown). This suggests that the IL-17A-supported granulomatous response in the absence of IL-27Ra is not mediated by neutrophil recruitment. However, because neutrophil accumulation during the chronic phase of Mtb infection is generally associated with susceptibility, ${ }^{76,77}$ the increased neutrophil levels might lead to the immunopathology observed in Mtb-infected IL-27Ra ${ }^{-1-}$ mice. ${ }^{17}$

In summary, our study gives evidence that an increased IL-17A secretion in the absence of IL-27Ra contributes to host resistance after Mtb infection and is associated with the accumulation of multifunctional $T$ cells and the strategic positioning of protective immune cells in the lung granuloma. These two components eventually support an optimal macrophage activation and improved elimination of Mtb. On a more systemic level, IL-27Ramediated signaling initially may prevent optimal protective immune responses by suppressing IL-17A production in Th17 cells. However, because uncontrolled IL-17A secretion is also associated with pathology in chronic inflammatory and autoimmune diseases, a tight control of this cytokine during Mtb infection is also ensured by IL-27Ra. Nevertheless, a timely inhibition of IL-27Ra-mediated signaling during vaccination or therapy may increase a coordinated protective effect of IL-17A without detrimental consequences.

\section{METHODS}

Mice

Breeding pairs of $\mathrm{IL}-27 \mathrm{Ra}^{-1-} 27$ and IL-17A ${ }^{-1-} 78$ mice on a C57BL/6 genetic background were kindly provided by Chris Saris (Amgen, CA) and Yoichiro Iwakura (University of Tokyo, Tokyo, Japan), respectively, and offspring were generated under specific pathogen-free conditions at the Research Center Borstel. To analyze the function of IL-17A in the absence of IL-27Ra-mediated 
a

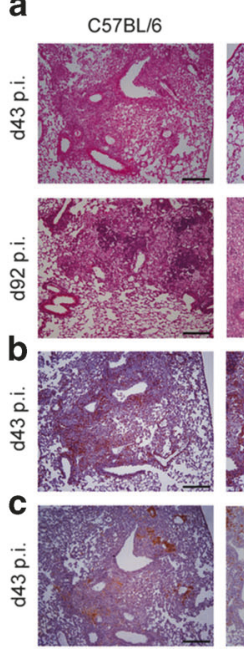

$\mathrm{IL}-27 \mathrm{R} \alpha^{-1-}$

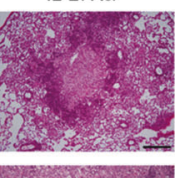

IL-27R $\alpha^{-1-}-1 \mathrm{LL}-17 \mathrm{~A}+1-$

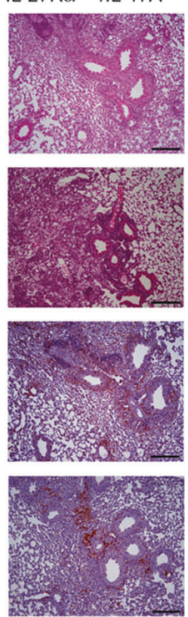

d

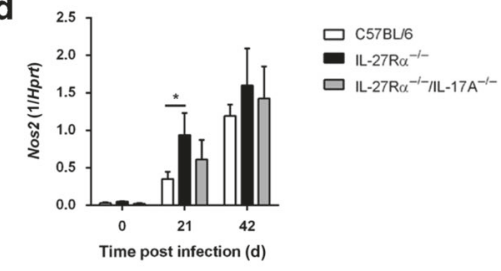

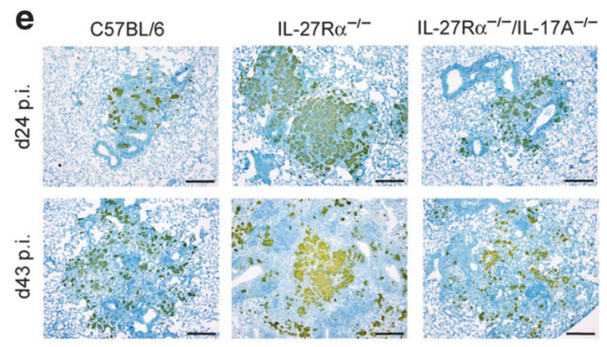

Fig. 9 IL-17A promotes the formation of highly stratified granulomas and NOS2 production in the lungs of Mtb-infected IL-27R $\alpha^{-1-}$ mice.

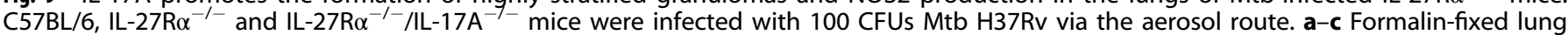
sections were stained with H\&E (a), for CD3 (b) or for B220 (c). Representative sections from 5 mice per group are shown and are from one of two representative experiments performed. Magnification 100x, bar, $200 \mu \mathrm{m}$. d The gene expression of Nos 2 in lung homogenates was quantified by real-time PCR. Data represent mean \pm SD of 5 mice per group and are from one of two representative experiments performed. Statistical analysis was performed using a two-way ANOVA with Bonferroni post hoc test defining differences as significant $\left({ }^{*} p<0.05\right)$. e Formalin-fixed lung sections were stained for NOS2 and counterstained with hematoxylin. Representative sections from 5 mice per group are shown and are from one of two representative experiments performed. Magnification 100x, bar, $200 \mu \mathrm{m}$

signaling, IL-27Ra ${ }^{-1-}$ mice were intercrossed with IL-17A ${ }^{-1-}$ animals (IL-27Ra ${ }^{-1-} / \mathrm{IL}-17 \mathrm{~A}^{-1-}$ ). As wild-type controls, C57BL/6 mice were purchased from Charles River. During infection experiments, mice were kept under barrier conditions in the BSL3 facility at the Research Center Borstel in individually ventilated cages. All experiments were conducted according to the German animal protection laws and were approved by the Animal Research Ethics Board of the Ministry of Environment (Kiel, Germany).

Bacteria and infection

If not otherwise stated, experimental mice were infected with Mtb H37Rv. Mtb HN878 was kindly provided by Reto Guler and Frank Brombacher (Cape Town, University of Cape Town, South Africa). To infect experimental animals, stock solutions of Mtb H37Rv or HN878 were diluted in sterile distilled water and pulmonary infection was performed using an inhalation exposure system (Glas-Col, Terre-Haute, IN). To infect mice with 100 CFU/lung, animals were exposed for $40 \mathrm{~min}$ to an aerosol generated by nebulizing $6.5 \mathrm{ml}$ of a suspension containing $10^{7}$ live bacteria. The inoculum size was checked $24 \mathrm{~h}$ after infection by determining the bacterial load in undiluted homogenates of the entire lung of infected mice.

Colony enumeration assay and disease scoring

Bacterial loads in the lungs were evaluated at different time points after infection. Lungs from killed animals were removed aseptically, weighed and homogenized in phosphate-buffered saline (PBS) containing a proteinase inhibitor cocktail (Roche Diagnostics, Mannheim, Germany) using the FastPrep ${ }^{\text {TM }}$ System (MP Biomedicals, Solon, USA). Tenfold serial dilutions of organ homogenates were plated onto Middlebrook $7 \mathrm{H} 10$ agar plates containing $10 \%$ oleic, albumin, dextrose and catalase (OADC). After incubation at $37^{\circ} \mathrm{C}$ for 21 days, colonies on plates were enumerated. All animals were weighed regularly before and after infection using a laboratory balance, and as a means for evaluating disease progression the body weight change was calculated.

\section{Histology}

One lung lobe per mouse was fixed in 4\% formalin-PBS, set in paraffin blocks and sectioned $(2 \mu \mathrm{m})$. Histopathological analyses were performed using standard protocols for hematoxylin/eosin staining. For the immunohistochemical detection of NOS2 and $\mathrm{CD} 3$, lung sections were deparaffinized and pressure cooked in 10 $\mathrm{mM}$ citrate buffer, $\mathrm{pH}$ 6. After peroxidase quenching with $1 \%$ $\mathrm{H}_{2} \mathrm{O}_{2}$ /tris-buffered saline (TBS) and blocking using the Avidin/ Biotin blocking kit (Vector Laboratories), and in the case of CD3 with $10 \%$ goat serum/TBS, sections were incubated with a primary rabbit-anti-NOS2 antibody (Merck Millipore) or rabbit-anti-CD3 antibody (Abcam) overnight followed by incubation with a biotinlabeled goat-anti-rabbit secondary antibody (Dianova, Hamburg, Germany). Development was performed by using the Vectastain Elite $A B C$ Kit (Vector, Burlingame, $C A$ ) and diaminobenzidin (Vector). For the immunohistochemical detection of CD45R/B220, deparaffinized sections were incubated with $1 \% \mathrm{H}_{2} \mathrm{O}_{2} / \mathrm{PBS}$ and blocked with $10 \%$ fetal calf serum (FCS)/PBS. Sections were incubated with a rat-anti-CD45R/B220 antibody overnight followed by the incubation with biotin-labeled goat-anti-rat secondary antibody. Development was performed by using the Vectastain Elite $A B C$ Kit (Vector, Burlingame, $C A$ ) and diaminobenzidin (Vector).

Preparation of single-cell suspensions from infected lungs For flow cytometric analysis, single-cell suspensions of lungs were prepared from Mtb-infected mice at different time points. Lungs were perfused through the right ventricle with warm PBS. Once lungs appeared white, they were removed and sectioned. Dissected lung tissue was then incubated in collagenase A $(0.7$ $\mathrm{mg} / \mathrm{ml}$, Roche Diagnostics, Mannheim, Germany) and DNase (30 $\mu \mathrm{g} / \mathrm{ml}$, Sigma) at $37^{\circ} \mathrm{C}$ for $2 \mathrm{~h}$. Digested lung tissue was gently disrupted by subsequent passage through a $100 \mu \mathrm{m}$ pore size nylon cell strainer. Suspensions were depleted from remaining erythrocytes using hypotonic red cell lysis buffer. Recovered vital lung cells were diluted in RPMI-1640 medium (Sigma) supplemented with $10 \%$ FCS (Life Technologies), $0.05 \mathrm{mM} \quad \beta$ mercaptoethanol (Sigma), and penicillin and streptomycin (100 
$\mathrm{U} / \mathrm{ml}$ and $100 \mu \mathrm{g} / \mathrm{ml}$; Life Technologies) and counted using an automatic cell counter $\left(\right.$ ViCell ${ }^{\circledR}$, Beckman Coulter, Krefeld, Germany).

Flow cytometry

For flow cytometric analysis of surface markers, single-cell suspensions of lungs were incubated with a mixture containing anti-FcyRIII/II antibody (Biolegend) as well as mouse, rat and hamster serum to block nonspecific binding. Cells were then incubated with optimal concentrations of the Mtb-specific IA $^{\mathrm{b}}$ ESAT6 $_{4-17}$-APC tetramer or the HLA-DQ2 CLIP $87-101-A P C$ control tetramer (kindly provided by the NIH Tetramer Core facilities, Emory University, Atlanta, GA), anti-CD90.2-APCeFluor780 (eBioscience), anti-CD44-FITC, anti-CD69-BV605, anti-PD1-PE-Cy7, anti-CD127-BV785 (all from Biolegend), antiCD4-V500, anti-CD62L-APC and anti-KLRG1-BV711 (all from BD Biosciences). For intracellular cytokine staining, $1 \times 10^{6}$ cells were stimulated with plate-bound anti-CD3/anti-CD28 (each 5 $\mu \mathrm{g} / \mathrm{ml}, \mathrm{BD}$ Bioscience) or ST-CF ${ }^{79}(10 \mu \mathrm{g} / \mathrm{ml})$ for $4.5 \mathrm{~h}$ in the presence of GolgiPlug ${ }^{\mathrm{TM}}$ (BD Biosciences). Cells were stained with optimal concentrations of anti-CD4-V500 (BD Biosciences), anti-CD44-FITC (Biolegend) and anti-CD90.2-APC-eFluor780 (eBioscience). Afterwards, cells were fixed and permeabilized with Cytofix/Cytoperm ${ }^{\text {TM }}$ (BD Biosciences). Intracellular accumulated cytokines were stained with anti-IFN- $\gamma-P E$, anti-TNF- $a-P E-$ Cy7 (both from Biolegend), anti-IL-17A-PerCP-Cy5.5 (eBioscience) and anti-IL-2-APC (BD Biosciences). For analysis of $\operatorname{Tr} 1$ cells, $2 \times 10^{6}$ cells were stimulated with plate-bound antiCD3/anti-CD28 (each $5 \mu \mathrm{g} / \mathrm{ml}, \mathrm{BD}$ Bioscience) or with phorbol 12-myristate 13-acetate (PMA) and ionomycin (each $0.5 \mu \mathrm{g} / \mathrm{ml}$, Sigma) for $4.5 \mathrm{~h}$ in the presence of GolgiPlug ${ }^{\mathrm{TM}}$ (BD Biosciences). Because PMA and ionomycin stimulation downregulates CD4 expression, $\mathrm{CD}^{+}{ }^{+} \mathrm{T}$ cells were characterized as CD8 negative and $T C R \beta / C D 3$ positive. For staining, the mouse regulatory T-cell staining kit (eBioscience) was used supplemented with the following antibodies: anti-CD8a-FITC (BD Biosciences), antiTCR $3-P E-C y 7$, anti-IL-10-PE (both from Biolegend) and antiFoxp3-eFluor450 (eBioscience). Fluorescence Minus One (FMO) staining was used as control. Data were acquired on a FACSCanto $^{T M}$ II (BD Bioscience) or on a LSRII (BD Bioscience) and analyzed with the FCS Express 4 Flow Cytometry software (DeNovo ${ }^{T M}$ Software).

ESAT6 $_{1-20}$-specific Elispot assays

Detection of antigen-specific IL-17A- or IFN- $\gamma$-producing cells from infected lungs was conducted using Elispot assay kits (R\&D Systems and BD Biosciences, respectively). To enrich CD4 ${ }^{+} \mathrm{T}$ cells, single-cell suspensions were incubated with magnetic CD4 MicroBeads (Miltenyi, Bergisch Gladbach, Germany) and separated from other cells using the autoMACS ${ }^{\circledR}$ Pro Separator (Miltenyi). Separated $\mathrm{CD}^{+} \mathrm{T}$ cells were collected in Iscove's modified Dulbecco's medium (Life Technologies) supplemented with $10 \%$ FCS (Life Technologies), $0.05 \mathrm{mM} \beta$-mercaptoethanol (Sigma), penicillin and streptomycin $(100 \mathrm{U} / \mathrm{ml}$ and $100 \mu \mathrm{g} / \mathrm{ml}$, Life Technologies) and counted. Cells were seeded in wells of antiIFN- $\gamma$ - or anti-IL-17A-coated MultiScreen HTS-IP filter plates $(1 \times$ $10^{5}$ cells/well) and twofold serial dilutions were made. MitomycinD-inactivated splenocytes from uninfected wild-type mice were used as antigen-presenting cells $\left(1 \times 10^{6}\right.$ cells/well). Cells were stimulated with ESAT6 ${ }_{1-20}(10 \mu \mathrm{g} / \mathrm{ml}$, Research Center Borstel) and recombinant IL-2 (10 U/ml, Peprotech, Hamburg, Germany) in 5\% $\mathrm{CO}_{2}$ at $37^{\circ} \mathrm{C}$. After $20 \mathrm{~h}$, anti-IFN- $\gamma$-coated plates were washed and a biotinylated anti-IFN- $\gamma$-antibody was used to detect the captured cytokine. Spots were visualized using streptavidin-horseradish peroxidase and AEC solution as a substrate. After $24 \mathrm{~h}$, anti-IL-17Acoated plates were washed and a biotinylated anti-IL-17Aantibody was used to detect the captured cytokine. Spots were visualized using streptavidin-AP and BCIP/NBT solution as a substrate. Spots were automatically enumerated using an Elispot reader (EliSpot $04 \mathrm{XL}$; AID).

\section{Quantitative real-time PCR}

Lung samples were homogenized in $4 \mathrm{M}$ guanidiniumisothiocyanate buffer and total RNA was extracted by acid phenol extraction. Complementary DNA was obtained using RevertAid $\mathrm{H}$ Minus M-MuLV reverse transcriptase (Fermentas) and random hexamer (Fermentas) as a primer. Quantitative PCR was performed on a Light $\mathrm{Cycler}^{\circledR} 480$ Instrument (Roche Diagnostics). Data were analyzed employing the "Second Derivative Maximum Method" and "Standard Curve Method" using Hprt as a housekeeping gene to calculate the level of gene expression in relation to Hprt. The following primer and probe sets were employed: Hprt: sense $5^{\prime}$ TCC TCC TCA GAC CGC TाT T-3', antisense 5'-CCT GGT TCA TCA TCG CTA ATC-3', probe 5'-AGT CCA G-3'; CCl20: sense 5'-GGT ACT GCT GGC TCA CCT CT-3', antisense $5^{\prime}$-TGT ACG AGA GGC AAC AGT CG-3', probe 5'-GGC AGA AG-3'; Cxc19: sense 5'-CTT TTC CTC TTG GGC ATC AT-3', antisense 5'-GCA TCG TGC ATT CCT TAT CA-3', probe 5'-CCT GGA GC-3'; CxCl10: sense 5'-GCT GCC GTC ATT TTC TGC- $3^{\prime}$, antisense $5^{\prime}$-TCT CAC TGG CCC GTC ATC- $3^{\prime}$, probe $5^{\prime}$-CTG CTG GG-3'; CxCl13: sense 5'-CAT AGA TCG GAT TCA AGT TAC GC-3', antisense 5'-CAC ACA TAT AAC TाT CTT CAT CTT GGT-3', probe 5'ATG GCT GC-3'; Nos2: sense 5'-CTT TGC CAC GGA CGA GAC-3', antisense $5^{\prime}$-TCA TTG TAC TCT GAG GGC TGA C-3', probe $5^{\prime}$-AGG CAG AG-3'.

\section{Delayed-type hypersensitivity}

At 35 days after infection, mice were challenged with an s.c. injection of $10 \mu \mathrm{g}$ of PPD (Statens Serum Institut, Copenhagen, Denmark) in $50 \mu \mathrm{l}$ of PBS in the right and $50 \mu \mathrm{l}$ of PBS in the left footpad. Swelling in both footpads was measured after $72 \mathrm{~h}$ using a Mitutoyo micrometer caliper (Brütsch), and the difference was taken as the amount of antigen-specific DTH.

Statistical analysis

Quantifiable data are expressed as the means of individual determinations and SDs. For the majority of experiments, a twoway analysis of variance (ANOVA) with Bonferroni post hoc test was used. For the comparison of groups at one time point, a oneway ANOVA with Bonferroni post hoc test was used. For $p$ values of $\leq 0.05$, results were considered statistically significant.

\section{ACKNOWLEDGEMENTS}

We are thankful to Chris Saris (Amgen, CA) and Yoichiro Iwakura (University of Tokyo, Tokyo, Japan) for providing IL-27Ra ${ }^{-1-}$ and IL-17A ${ }^{-1-}$ mice, respectively. We are also grateful to Marion Schuldt and Kerstin Traxel for supplying and cleaning the lab and to Ika Monath and Christine Keller for organizing the animal facility and taking care of the mice. The $I \mathrm{~A}^{\mathrm{b}} \mathrm{ESAT6}_{4-17}$ and control tetramers were kindly provided by the $\mathrm{NIH}$ Tetramer Core facilities, Emory University, Atlanta, GA. This work was supported by the Cluster of Excellence Inflammation-at-Interfaces (EXC306).

\section{AUTHOR CONTRIBUTIONS}

$\mathrm{HE}$, JB and $\mathrm{CH}$ designed the study; $\mathrm{HE}$, JB and KR performed the experiments and analyzed the results; $\mathrm{AH}$ and JV performed the experiments; IR generated reagents; $\mathrm{HE}, \mathrm{JB}, \mathrm{KR}$ and $\mathrm{CH}$ drafted the figures and manuscript.

\section{ADDITIONAL INFORMATION}

Competing interests: The authors declare no competing interests.

Publisher's note: Springer Nature remains neutral with regard to jurisdictional claims in published maps and institutional affiliations. 


\section{REFERENCES}

1. WHO. Global Tuberculosis Report - 2016 (WHO, Geneva, Switzerland, 2016),

2. Saunders, B. M. \& Britton, W. J. Life and death in the granuloma: immunopathology of tuberculosis. Immunol. Cell Biol. 85, 103-111 (2007).

3. Lin, Y. et al. Interleukin-17 is required for $\mathrm{T}$ helper 1 cell immunity and host resistance to the intracellular pathogen Francisella tularensis. Immunity $\mathbf{3 1}$, 799-810 (2009).

4. Roach, D. R. et al. TNF regulates chemokine induction essential for cell recruitment, granuloma formation, and clearance of mycobacterial infection. J. Immunol. 168, 4620-4627 (2002).

5. Scott Algood, H. M., Lin, P. L. \& Flynn, J. L. Tumor necrosis factor and chemokine interactions in the formation and maintenance of granulomas in tuberculosis. Clin. Infect. Dis. 41, S189-S193 (2005).

6. Geldmacher, C., Zumla, A. \& Hoelscher, M. Interaction between HIV and Mycobacterium tuberculosis: HIV-1-induced CD4 T-cell depletion and the development of active tuberculosis. Curr. Opin. HIV AIDS 7, 268-274 (2012).

7. Havlir, D. V. \& Barnes, P. F. Tuberculosis in patients with human immunodeficiency virus infection. New Engl. J. Med. 340, 367-373 (1999).

8. Flory, C. M., Hubbard, R. D. \& Collins, F. M. Effects of in vivo T lymphocyte subset depletion on mycobacterial infections in mice. J. Leukoc. Biol. 51, 225-229 (1992).

9. Leveton, C. et al. T-cell-mediated protection of mice against virulent Mycobacterium-tuberculosis. Infect. Immun. 57, 390-395 (1989).

10. Pearl, J. E., Saunders, B., Ehlers, S., Orme, I. M. \& Cooper, A. M. Inflammation and lymphocyte activation during mycobacterial infection in the interferon- $\hat{l}^{3}$ deficient mouse. Cell. Immunol. 211, 43-50 (2001).

11. Elias, D., Akuffo, H. \& Britton, S. PPD induced in vitro interferon gamma production is not a reliable correlate of protection against Mycobacterium tuberculosis. Trans. R. Soc. Trop. Med. Hyg. 99, 363-368 (2005).

12. Darrah, P. A. et al. Multifunctional TH1 cells define a correlate of vaccinemediated protection against Leishmania major. Nat. Med. 13, 843-850 (2007).

13. Wille-Reece, U. et al. Toll-like receptor agonists influence the magnitude and quality of memory $T$ cell responses after prime-boost immunization in nonhuman primates. J. Exp. Med. 203, 1249-1258 (2006).

14. Aagaard, C. et al. Protection and polyfunctional T cells induced by Ag85B-TB10.4/ IC31 against Mycobacterium tuberculosis is highly dependent on the antigen dose. PLoS One 4, e5930-e5930 (2009).

15. Derrick, S. C., Yabe, I. M., Yang, A. \& Morris, S. L. Vaccine-induced anti-tuberculosis protective immunity in mice correlates with the magnitude and quality of multifunctional CD4 T cells. Vaccine 29, 2902-2909 (2011).

16. Lindenström, T. et al. Tuberculosis subunit vaccination provides long-term protective immunity characterized by multifunctional CD4 memory T cells. J. Immunol. 182, 8047-8055 (2009).

17. Hölscher, C. et al. The IL-27 receptor chain WSX-1 differentially regulates antibacterial immunity and survival during experimental tuberculosis. J. Immunol. 174, 3534-3544 (2005).

18. Pearl, J. E. et al. IL-27 signaling compromises control of bacterial growth in mycobacteria-infected mice. J. Immunol. 173, 7490-7496 (2004).

19. Pflanz, S. et al. IL-27, a heterodimeric cytokine composed of EBI3 and p28 protein induces proliferation of naive CD4(+) T cells. Immunity 16, 779-790 (2002).

20. Liu, J., Guan, X. \& Ma, X. Regulation of IL-27 p28 gene expression in macrophages through MyD88- and interferon-1̂̉â "mediated pathways. J. Exp. Med. 204, 141-152 (2007).

21. Pirhonen, J., Siren, J., Julkunen, I. \& Matikainen, S. IFN-alpha regulates Toll-like receptor-mediated IL-27 gene expression in human macrophages. J. Leukoc. Biol. 82, 1185-1192 (2007).

22. Zhang, J. et al. Activation of IL-27 p28 gene transcription by interferon regulatory factor 8 in cooperation with interferon regulatory factor 1. J. Biol. Chem. 285 21269-21281 (2010).

23. Pflanz, S. et al. WSX-1 and glycoprotein 130 constitute a signal-transducing receptor for IL-27. J. Immunol. 172, 2225-2231 (2004).

24. Chen, Q. et al. Development of Th1-type immune responses requires the type cytokine receptor TCCR. Nature 407, 916-920 (2000).

25. Hibbert, L., Pflanz, S., de Waal Malefyt, R. \& Kastelein, R. A. IL-27 and IFN-alpha signal via Stat 1 and Stat3 and induce T-Bet and IL-12Rbeta2 in naive T cells. J. Interferon Cytokine Res. 23, 513-522 (2003).

26. Takeda, A. et al. Cutting edge: role of IL-27/WSX-1 signaling for induction of T-bet through activation of STAT1 during initial Th1 commitment. J. Immunol. 170, 4886-4890 (2003).

27. Yoshida, $H$. et al. WSX-1 is required for the initiation of Th1 responses and resistance to L. major infection. Immunity 15, 569-578 (2001).

28. Artis, D. et al. The IL-27 receptor (WSX-1) is an inhibitor of innate and adaptive elements of type 2 immunity. J. Immunol. 173, 5626-5634 (2004).
29. Batten, M. et al. Interleukin 27 limits autoimmune encephalomyelitis by suppressing the development of interleukin 17-producing T cells. Nat. Immunol. 7 929-936 (2006).

30. Villarino, A. et al. The IL-27R (WSX-1) is required to suppress T cell hyperactivity during infection. Immunity 19, 645-655 (2003).

31. Apetoh, L. et al. The aryl hydrocarbon receptor interacts with c-Maf to promote the differentiation of type 1 regulatory T cells induced by IL-27. Nat. Immunol. 11, 854-861 (2010).

32. Pot, C. et al. Cutting edge: IL-27 induces the transcription factor c-Maf, cytokine IL-21, and the costimulatory receptor ICOS that coordinately act together to promote differentiation of IL-10-producing Tr1 cells. J. Immunol. 183, 797-801 (2009).

33. Owaki, T. et al. IL-27 suppresses CD28-medicated IL-2 production through suppressor of cytokine signaling 3. J. Immunol. 176, 2773-2780 (2006).

34. Lucas, S., Ghilardi, N., Li, J. \& de Sauvage, F. J. IL-27 regulates IL-12 responsiveness of naive CD4+T cells through Stat1-dependent and -independent mechanisms. Proc. Natl. Acad. Sci. USA 100, 15047-15052 (2003).

35. Diveu, C. et al. IL-27 blocks RORc expression to inhibit lineage commitment of Th17 cells. J. Immunol. 182, 5748-5756 (2009).

36. Heinemann, C. et al. IL-27 and IL-12 oppose pro-inflammatory IL-23 in CD4 +T cells by inducing Blimp1. Nat. Commun. 5, 3770 (2014).

37. Torrado, E. et al. Interleukin $27 \mathrm{R}$ regulates $\mathrm{CD} 4+\mathrm{T}$ cell phenotype and impacts protective immunity during Mycobacterium tuberculosis infection. J. Exp. Med. 212, 1449-1463 (2015).

38. Reiley, W. W. et al. Distinct functions of antigen-specific CD4 T cells during murine Mycobacterium tuberculosis infection. Proc. Natl. Acad. Sci. USA 107, 19408-19413 (2010).

39. Jin, W. \& Dong, C. IL-17 cytokines in immunity and inflammation. Emerg. Microbes Infect. 2, e60-e60 (2013).

40. Cho, J. S. et al. IL-17 is essential for host defense against cutaneous Staphyloccocus aureus infection in mice. J. Clin. Investig. 120, 1762-1773 (2010).

41. Ishigame, $H$. et al. Differential roles of interleukin-17A and $-17 \mathrm{~F}$ in host defense against mucoepithelial bacterial infection and allergic responses. Immunity $\mathbf{3 0}$ 108-119 (2009).

42. Ye, P. et al. Requirement of interleukin 17 receptor signaling for lung CXC chemokine and granulocyte colony-stimulating factor expression, neutrophil recruitment, and host defense. J. Exp. Med. 194, 519-527 (2001).

43. Erdmann, $\mathrm{H}$. et al. IL-17A promotes macrophage effector mechanisms against Trypanosoma cruzi by trapping parasites in the endolysosomal compartment. Immunobiology 218, 910-923 (2013).

44. Gaffen, S. L. Role of IL-17 in the pathogenesis of rheumatoid arthritis. Curr. Rheumatol. Rep. 11, 365-370 (2009).

45. Harper, E. G. et al. Th17 cytokines stimulate CCL20 expression in keratinocytes in vitro and in vivo: implications for psoriasis pathogenesis. J. Invest Dermatol. 129, 2175-2183 (2010).

46. Komiyama, Y. et al. IL-17 plays an important role in the development of experimental autoimmune encephalomyelitis. J. Immunol. 177, 566-573 (2006).

47. Park, H. et al. A distinct lineage of CD4 T cells regulates tissue inflammation by producing interleukin 17. Nat. Immunol. 6, 1133-1141 (2005).

48. Yihua, C. et al. Pivotal role of dermal IL-17-producing $\hat{~}^{3} \hat{I}^{\prime}$ T cells in skin inflammation. Immunity 35, 596-610 (2011).

49. Khader, S. A. et al. IL-23 compensates for the absence of IL-12p70 and is essential for the IL-17 response during tuberculosis but is dispensable for protection and antigen-specific IFN-gamma responses if IL-12p70 is available. J. Immunol. 175, 788-795 (2005).

50. Scriba, T. J. et al. Distinct, specific IL-17- and IL-22-producing CD4+T cell subsets contribute to the human anti-mycobacterial immune response. J. Immunol. 180, 1962-1970 (2008).

51. Gopal, R. et al. Interleukin-17-dependent CXCL13 mediates mucosal vaccineinduced immunity against tuberculosis. Mucosal Immunol. 6, 972-984 (2013).

52. Gopal, R. et al. Unexpected role for IL-17 in protective immunity against hypervirulent Mycobacterium tuberculosis HN878 infection. PLoS Pathog. 10, e1004099 (2014).

53. Khader, S. A. et al. IL-23 and IL-17 in the establishment of protective pulmonary $\mathrm{CD} 4+\mathrm{T}$ cell responses after vaccination and during Mycobacterium tuberculosis challenge. Nat. Immunol. 8, 369-377 (2007).

54. Hirota, K. et al. Preferential recruitment of CCR6-expressing Th17 cells to inflamed joints via CCL20 in rheumatoid arthritis and its animal model. J. Exp. Med. 204, 2803-2812 (2007).

55. Kim, C. H. Migration and function of Th17 cells. Inflamm. Allergy Drug Targets 8 , 221-228 (2009).

56. Chen, J. et al. The RNA-binding protein HuR contributes to neuroinflammation by promoting C-C chemokine receptor 6 (CCR6) expression on Th17 cells. J. Biol. Chem. 292, 14532-14543 (2017). 
57. Turner, J. E. et al. CCR6 recruits regulatory T cells and Th17 cells to the kidney in glomerulonephritis. J. Am. Soc. Nephrol. 21, 974-985 (2010).

58. Lee, A. Y. \& Korner, H. CCR6 and CCL20: emerging players in the pathogenesis of rheumatoid arthritis. Immunol. Cell Biol. 92, 354-358 (2014).

59. Okamoto Yoshida, Y. et al. Essential role of IL-17A in the formation of a mycobacterial infection-induced granuloma in the lung. J. Immunol. 184, 4414-4422 (2010).

60. Stumhofer, J. S. et al. Interleukin 27 negatively regulates the development of interleukin 17-producing $T$ helper cells during chronic inflammation of the central nervous system. Nat. Immunol. 7, 937-945 (2006).

61. Lockhart, E., Green, A. M. \& Flynn, J. L. IL-17 production is dominated by gammadelta $\mathrm{T}$ cells rather than CD4 T cells during Mycobacterium tuberculosis Infection. J. Immunol. 177, 4662-4669 (2006).

62. Happel, K. I. et al. Pulmonary interleukin-23 gene delivery increases local T-cell immunity and controls growth of Mycobacterium tuberculosis in the lungs. Infect. Immun. 73, 5782-5788 (2005).

63. Sakai, S., Kauffman, K. D., McBerry, C. C. \& Barber, D. L. Control of Mycobacterium tuberculosis infection by a subset of lung parenchyma homing CD4 T cells. J. Immunol. 192, 2965-2969 (2015).

64. Palma, C. et al. The Ag85B protein of Mycobacterium tuberculosis may turn a protective immune response induced by Ag85B-DNA vaccine into a potent but nonprotective Th 1 immune response in mice. Cell. Microbiol. 9, 1455-1465 (2007).

65. Huaman, M. C., Mullen, G. E. D., Long, C. A. \& Mahanty, S. Plasmodium falciparum apical membrane antigen 1 vaccine elicits multifunctional CD4 cytokineproducing and memory T cells. Vaccine 27, 5239-5246 (2009).

66. Seder, R. A., Darrah, P. A. \& Roederer, M. T-cell quality in memory and protection: implications for vaccine design. Nat. Rev. Immunol. 8, 247-258 (2008).

67. Sa, Khader et al. In a murine tuberculosis model, the absence of homeostatic chemokines delays granuloma formation and protective immunity. J. Immunol. 183, 8004-8014 (2009).

68. Rangel-Moreno, J. et al. The development of inducible bronchus-associated lymphoid tissue depends on IL-17. Nat. Immunol. 12, 639-646 (2011).
69. Schreiber, T. et al. Selectin ligand-independent priming and maintenance of T cell immunity during airborne tuberculosis. J. Immunol. 176, 1131-1140 (2006).

70. Kahnert, A. et al. Mycobacterium tuberculosis triggers formation of lymphoid structure in murine lungs. J. Infect. Dis. 195, 46-54 (2007).

71. Tsai, M. C. et al. Characterization of the tuberculous granuloma in murine and human lungs: Cellular composition and relative tissue oxygen tension. Cell Microbiol. 8, 218-232 (2006).

72. Ulrichs, T. et al. Human tuberculous granulomas induce peripheral lymphoid follicle-like structures to orchestrate local host defence in the lung. J. Pathol. 204, 217-228 (2004).

73. Bosio, C. M., Gardner, D. \& Elkins, K. L. Infection of B cell-deficient mice with CDC 1551 , a clinical isolate of Mycobacterium tuberculosis: delay in dissemination and development of lung pathology. J. Immunol. 164, 6417-6425 (2000).

74. Maglione, P. J., Xu, J. \& Chan, J. B. Cells moderate inflammatory progression and enhance bacterial containment upon pulmonary challenge with Mycobacterium tuberculosis. J. Immunol. 178, 7222-7234 (2007).

75. Seiler, P. et al. Early granuloma formation after aerosol Mycobacterium tuberculosis infection is regulated by neutrophils via CXCR3-signaling chemokines. Eur. J. Immunol. 33, 2676-2686 (2003).

76. Eruslanov, E. B. et al. Neutrophil responses to Mycobacterium tuberculosis infection in genetically susceptible and resistant mice neutrophil responses to Mycobacterium tuberculosis infection in genetically susceptible and resistant Mice. Infect. Immun. 73, 1744-1753 (2005).

77. Keller, C. et al. Genetically determined susceptibility to tuberculosis in mice causally involves accelerated and enhanced recruitment of granulocytes. Infect. Immun. 74, 4295-4309 (2006).

78. Nakae, S. et al. Antigen-specific T cell sensitization is impaired in IL-17-deficient mice, causing suppression of allergic cellular and humoral responses. Immunity 17, 375-387 (2002).

79. Rosenkrands, I. \& Andersen, P. Preparation of culture filtrate proteins from Mycobacterium tuberculosis. Methods Mol. Med. 54, 205-215 (2001). 